\title{
A MULTIDIMENSIONAL AND MULTISCALE MODEL FOR PRESSURE ANALYSIS IN A RESERVOIR-PIPE-VALVE SYSTEM
}

\author{
Feng Jie Zheng ${ }^{\text {a }}$, Chao Yong Zong ${ }^{\mathrm{a}}$, William Dempster ${ }^{\mathrm{b}}$, Fu Zheng Qu ${ }^{\mathrm{a}}$, Xue Guan Song ${ }^{\text {a }}$ \\ School of Mechanical Engineering, Dalian University of Technology, Dalian, Liaoning, China ${ }^{\text {a }}$ \\ Department of Mechanical and Aerospace Engineering, University of Strathclyde, Glasgow, G1 1XJ, UK ${ }^{\text {b }}$
}

[DOI: 10.1115/1.4044117]

\section{ABSTRACT}

Reservoir-pipe-valve (RPV) systems are widely used in many industrial processes. The pressure in an RPV system plays an important role in the safe operation of the system, especially during the sudden operations such as rapid valve opening or closing. To investigate the pressure response, with particular interest in the pressure fluctuations in an RPV system, a multidimensional and multiscale model combining the method of characteristics (MOC) and computational fluid dynamics (CFD) method is proposed. In the model, the reservoir is modeled as a zero-dimensional virtual point, the pipe is modeled as a one-dimensional system using the MOC, and the valve is modeled using a three-dimensional CFD model. An interface model is used to connect the multidimensional and multiscale model. Based on the model, a transient simulation of the turbulent flow in an RPV system is conducted, in which not only the pressure fluctuation in the pipe but also the detailed pressure distribution in the valve is obtained. The results show that the proposed model is in good agreement when compared with a high fidelity CFD model used to represent both large-scale and small-scale spaces. As expected, the proposed model is significantly more computationally efficient than the CFD model. This demonstrates the feasibility of analyzing complex RPV systems within an affordable computational time. 


\section{INTRODUCTION}

A reservoir-pipe-valve (RPV) system is a common configuration for many industrial processes found in industries such as gas and petroleum, food manufacturing, chemical and plastic manufacturing, and nuclear power. An RPV system mainly consists of three parts: (1) a reservoir is created to provide the storage and supply of fluid; (2) a pipe to convey the fluid; and 3) a valve to regulate the fluid flow by changing the passageways of the flow. However, an RPV system may malfunction, resulting in damage and uncontrolled discharge due to excessive pressure fluctuations induced by the operation of the valves.

Many investigations have been performed to analyze the pressure behavior of an RPV system by using numerical methods such as the method of characteristics (MOC) and Computational Fluid Dynamics (CFD). In the late 20th century, the development of computers promoted the application of MOC for the pressure analysis of large-scale RPV systems [1]. Tijsseling et al. [2-5] adopted MOC methods to investigate water hammer phenomenon in liquidfilled pipe systems and the axial vibration of liquid-filled pipes caused by fluid-structure interactions (FSI). Johnston et al. [6] introduced a mechanical damping-based conceptual transient model that considered the mechanical damping caused by the interaction of pipes and joints with surrounding soils. Xu and Jiao [7] proposed an improved MOC method using time-line interpolation rather than a recursive algorithm to speed-up the calculation. Ferràs et al. [8] analyzed the FSI physical phenomenon based on the implementation of a 1-D 4-equation MOC solver, which took into account dry friction arising from the shear between the pipe-wall and its surroundings. It has been verified that MOC is an efficient and reliable method to simulate pressure wave effects in RPV systems. However, the assumptions and/or simplifications implicit in the use of MOC require that all the boundaries (especially in some key fittings) can be expressed as simple time based functions and reduction of the entire RPV system to a 1-D representation. As a result, it is impossible to obtain the detailed flow field in the key fittings, such as the valve, which is a primary source of the pressure fluctuations. Thus, MOC inadequately predicts the pressure response of the whole RPV system.

CFD methods can overcome many of the short comings of the MOC. CFD analysis enables the investigation of 
the dynamic characteristics of crucial components with complex geometry and complicated boundaries, such as pumps, turbines and valves [9-15]. Song et al. [9-11] presented a series of techniques to model the motion of a valve disc within a CFD simulation of a spring-loaded pressure safety valve (PSV) which was used to investigate the dynamic characteristics of the valve and the operation conditions of the pressure vessel. Yang et al. [12] established a full-scale CFD model for the analysis of a large PSV used in nuclear power plants and confirmed that the use of CFD analysis can improve the design and operation of a pressure relief valve, particular for high temperature and high pressure conditions where testing is difficult to conduct. Although a detailed simulation of a valve operation is possible, a transient high fidelity simulation remains time consuming and may take a number of days. Since a thorough dynamic analysis or an optimization of a simple RPV system often requires a series of transient simulations, the whole process will usually take days to months, which may make the dynamic analysis or the optimization of RPV systems based on transient simulation impracticable for everyday engineering design use.

The determination of pressure fluctuations in an RPV system is a transient problem at a system level. Pressure waves propagating in the pipeline will couple strongly with the pressure variations caused by the disturbances of the upstream/downstream boundaries. Thus, it is less effective to investigate this problem using only the MOC or only the CFD method. However, a hybrid approach making use of each technique is likely to be a more efficient approach [16-20]. Yang et al. [16] applied this coupling method to study the dynamic characteristics of a pump response to transient events. Recently, a 1-D/3-D coupling was presented by Wang et al. [17] who simulated compressible water hammer in a hydraulic system, where a 1D MOC code is implemented as a set of boundary conditions in the OpenFOAM open source finite volume CFD software which accounted for the 3-D effects of relatively simple test case geometries. Mandair et al. [18] compared the MOC method with a full CFD simulation for a very simplified case involving the water hammer caused by the rapid closure of a knife edge valve, and compared the results with a water hammer experiment. Some other researchers [19-20] applied this coupling approach to gas service. All of these studies indicate that the hybrid coupling approach can improve accuracy at the coupling interfaces for relatively 
simple geometries. In this paper, we examine the application of a MOC/CFD coupled approach for a more complex and realistic valve geometries; in this case the 3-D flow and pressures fluctuations resulting from a ball valve closure.

Therefore, a multidimensional and multiscale model is proposed where the MOC and CFD method are combined and embedded within the FLUENT [21] simulation platform using its User Defined Function (UDF) capability, thus mitigating the need for external compilers. Based on this MOC-CFD model, a transient simulation of the turbulent flow in an RPV system is conducted, where the reservoir and the pipeline are simplified as a virtual pressure source and virtual line element, respectively. The remaining sections of the pipe and valve are evaluated using a 3-D CFD model. This method is validated by comparison with a complete representation of the RPV system using CFD simulation. The improvement in the modelling approach suggested by this paper is realized in a number of ways. Principally, taking the velocity profile of the moving valve interface provides a more accurate technique for pressure wave generation. Implementation of an accurate MOC model is also investigated through comparison with a full CFD solution. Specifically, a more realistic wave speed of the water is used instead of an integer that is rounded off to suit the pipe length, and different friction models were evaluated in the MOC and MOC-CFD models.

The paper is structured as follows: the implementation of the proposed model is described first, then the validation of the model using a complete CFD model is discussed and finally the application of the technique is presented by assessing the pressure wave propagation under some typical conditions.

\section{NUMERICAL MODEL}

In an RPV system, a valve is an active component where the flow change induced by its opening/closing will result in a series of pressure waves which will propagate through the pipeline and reflect at both ends of the system until the energy is dissipated. In many previous studies of an RPV system, the valve is assumed to be opened/closed suddenly or with a simple operational pattern because the resulting complex flow field due to the valve operation is difficult to describe with a simplified model, and the large scale modelling requirements of the system is timeconsuming when performing the simulation using a CFD model. In this section, a multidimensional and multiscale 
model combining MOC and the CFD method (MOC-CFD model) is established to explore the transient characteristics of the RPV system at a system level.

\subsection{Compressible model}

To observe the propagation of the pressure waves in the pipeline, the fluid medium is compressible in the derivation of the governing equations, while the pipe wall is considered to be rigid. Thus, the pressure wave velocity $a$ can be expressed as follows:

$$
a=\sqrt{\frac{K}{\rho}}
$$

where $\rho$ is the mass density of the fluid, and $K$ is the fluid bulk modulus of elasticity $\left(2.2 \times 10^{9} \mathrm{~Pa}\right.$ at $25^{\circ} \mathrm{C}$ and 1 Atm) defined as the ratio of the infinitesimal pressure increase to the resulting relative decrease of the volume .

$$
K=\frac{\mathrm{d} P}{\mathrm{~d} \rho / \rho}
$$

in which $P$ is the pressure.

When the reference pressure $P_{0}$, set as the atmospheric pressure, the corresponding density $\rho_{0}$ can be obtained easily and the pressure wave velocity is $a_{0}=\sqrt{\frac{K}{\rho_{0}}}$. The density variation can be derived from equation (2):

$$
\rho=\frac{\rho_{0}}{\left(1.0-\frac{P-P_{0}}{K}\right)}
$$

The equations presented above will be prescribed in the CFD simulation to capture the effects of the pressure waves in an RPV system, including the flow field adjacent to the valve. However, if the pipeline is modeled using a MOC solution method, it is computationally expensive to account for the variation of $\rho$ and $a$. As can be seen from Eq. (1-2), the values of the variations of $P$ and $\rho$ differ by six orders of magnitude (here $\rho$ is about 1000 $\mathrm{kg} / \mathrm{m}^{3}$ ). Thus, a pressure change of $1.0 \mathrm{MPa}$ leads to a discrepancy in the density of water by about $0.1 \%$ and a change of pressure wave velocity of about $0.05 \%$.

2.2 MOC theory 
2.2.1 The basic theory of MOC [22]

The inlet pipe is assumed to be a 1-D model, and the continuity and momentum equations for transient pipe flow are [22]:

$$
\begin{gathered}
\frac{\partial P}{\partial t}+V \frac{\partial P}{\partial x}+\rho a^{2} \frac{\partial V}{\partial x}=0 \\
\frac{\partial V}{\partial t}+V \frac{\partial V}{\partial x}+\frac{1}{\rho} \frac{\partial P}{\partial x}+\frac{f(t)}{\rho}=0
\end{gathered}
$$

where the pressure $P$ and the mean sectional velocity $V$ in the pipeline are functions of the flow time $t$ and the pipe length $x$, and $f(t)$ is the friction model, as discussed in Section 2.2.2. Here, the convective acceleration terms, $V \frac{\partial V}{\partial x}$ and $V \frac{\partial P}{\partial x}$ are negligible because they are small compared to the other terms [22].

Consider a linear combination of equations (4) and (5) with the total derivatives of the variables expressed as follows:

$$
\begin{aligned}
& \frac{d V}{d t}=\frac{\partial V}{\partial t}+\frac{\partial V}{\partial x} \frac{d x}{d t} \\
& \frac{d P}{d t}=\frac{\partial P}{\partial t}+\frac{\partial P}{\partial x} \frac{d x}{d t}
\end{aligned}
$$

The compatibility equations can be written as follows:

$$
\begin{gathered}
C+\left\{\begin{array}{l}
\frac{d V}{d t}+\frac{1}{\rho a} \frac{d P}{d t}+\frac{f(t)}{\rho}=0 \\
\frac{d x}{d t}=a
\end{array}\right. \\
C+\left\{\begin{array}{l}
\frac{d V}{d t}-\frac{1}{\rho a} \frac{d P}{d t}+\frac{f(t)}{\rho}=0 \\
\frac{d x}{d t}=-a
\end{array}\right.
\end{gathered}
$$

The partial differential equations (equations (4) and (5)) are converted into ordinary differential equations (equations (8) and (9)) along specific lines, $\mathrm{C}+$ and $\mathrm{C}-$, as shown in Fig. 1 (these are called characteristic lines). These characteristic lines represent the traveling path of fluctuations initiated at various locations in the system, where the interval between two adjacent cross sections is $\Delta x=a \Delta t$. 


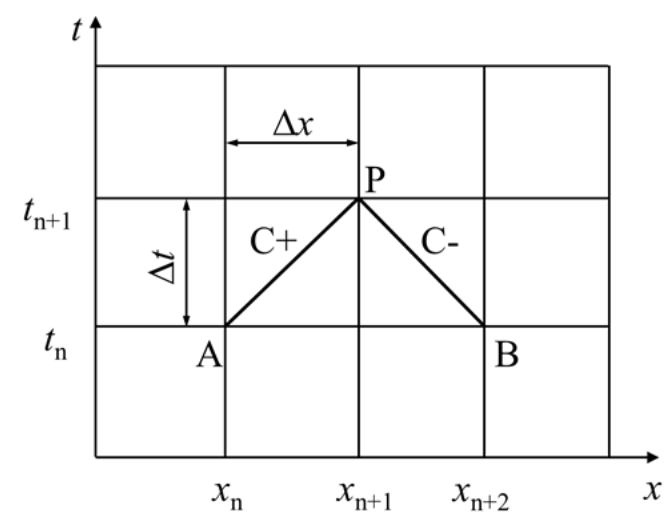

Fig. 1 Characteristic lines in $\mathrm{x}$-t plane

Integrating the first equations of equation (8) and (9) along the characteristic lines, we obtain

$$
\begin{cases}C+ & V_{P}=C_{p}-C_{a} P_{P} \\ C- & V_{P}=C_{n}+C_{a} P_{P}\end{cases}
$$

where

$$
\left\{\begin{array}{l}
C_{p}=V_{A}+\frac{P_{A}}{\rho a}-\frac{A f_{A}(t)}{\rho} \\
C_{n}=V_{B}-\frac{P_{B}}{\rho a}-\frac{A f_{B}(t)}{\rho} \\
C_{a}=\frac{1}{\rho a}
\end{array}\right.
$$

in which $A$ is the cross-sectional area of the pipe flow, $f_{A}(t)$ and $f_{B}(t)$ are the friction terms with respect to the flow characteristics at points $\mathrm{A}$ and $\mathrm{B}$, respectively.

\subsubsection{Friction model}

In normal practice, pipe friction generated during transient flow is approximately calculated using the steady friction model (SFM), which is based on the average velocity of the fluid flow.

$$
f_{S F M}(t)=\frac{f_{0} \rho V^{2}}{2 D}
$$

where $f_{0}$ is the Darcy-Weisbach friction factor, and $D$ is the pipe diameter. 
However, the energy losses during the transient state are not the same as those for steady flow. This poses serious limitations for predicting the pressure surges of fast transients induced by the quick closure of a downstream valve.

To address this issue, several unsteady friction models have been proposed with the development of transient flow theory. Most of those models are 1-D models because the more accurate quasi-2-D model [23] is computationally expensive and thus is only suitable for simple RPV systems. Therefore, two types of 1-D transient friction models are used and evaluated in this paper: an instantaneous acceleration-based model (IABM) and a convolution integral model (CIM).

IABM is based on an empirical assumption that the energy dissipation is attributed to instantaneous local and convective accelerations. The most used IABM, which was proposed by Brunone et al. [24], takes both the local inertia and the friction forces in fast transients into account through an addition to the preceding SFM; its expression is:

$$
f_{I B A M}(t)=\frac{f_{0} \rho V^{2}}{2 D}+k \rho\left(\frac{\partial V}{\partial t}-a \frac{\partial V}{\partial x}\right)
$$

where $k$ is the Brunone decay coefficient and is independent of time and space for sake of simplicity. The above IABM is a one-coefficient model $(k)$, however, two-coefficient models [25] have been developed that consider the local and convective accelerations independently. For one or two-coefficient IABM approaches, the decay coefficients are obtained through experiments. In this paper, a one-coefficient IABM (equation (13)) is used to account for the pressure dissipation in transient flow.

An alternative one-dimensional model, CIM, was first introduced by Zielke [26]. CIM is based on deriving an exact solution for the frequency dependent friction factor (highly dependent upon the rate of the change of the velocity) for laminar flow, which relates the energy dissipation in transient laminar flow to the instantaneous mean velocity and the past velocity changes. It can be expressed as: 


$$
f_{C I M}(t)=\frac{f_{0} \rho V^{2}}{2 D}+\frac{16 \rho v}{D^{2}} \int_{0}^{t} \frac{\partial V}{\partial t}(u) W(t-u) d u
$$

where $u$ is a dummy variable that physically represents the instantaneous time in the time history, and $v$ is the kinematic viscosity of the fluid. $W$ is a weighting for past velocity changes that is obtained from an inverse Laplace transformation and can be approximately expressed as follows:

$$
W(\tau) \approx W_{1}(\tau)+W_{2}(\tau)+W_{3}(\tau)
$$

where $W_{i}(\tau)=m_{i} e^{-n_{i} \tau}$ (for $i=1,2,3$ ) and the non-dimensional time $\tau=\frac{4 v}{D^{2}} t$.

The convolution integral requires much more computer memory with the progression of time and combined with the time-dependent weighting function, the finite difference schemes deploying CIM are found to be time consuming. To address this issue, Trikha [27] simplified Zielke's model, reduced the required computing power and extended the CIM approach to turbulent unsteady flow; however, its accuracy is reduced at the same time. Building on Zielke's model, Vardy et al. [28,29] established a weighting function model that is related to the non-dimensional time $\tau$ as well as the product of $f_{0}$ and the Reynolds number $R e$; this new weighting model (based on an approximation of a turbulent pipe flow as a laminar annulus surrounding a uniform core) is substituted into Eq. (14) in this paper.

The friction factor in turbulent flow can be simplified as follows [30]:

$$
\sqrt{\frac{1}{f_{0}}}=-1.8 \log _{10}\left(\frac{6.9}{\operatorname{Re}}\right)
$$

The friction models mentioned in equations (12-14) will be subsequently evaluated.

\subsection{Mathematical basis for the CFD model [31]}

Since the 1990s, CFD has been widely used in the industrial community, because of the availability of highperformance computing hardware and user-friendly interfaces. The mathematical basis for a comprehensive model of fluid flow utilizes the basic principles of conservation of mass, momentum, energy and the equation of state, whose 
most general form are either integral equations or partial differential equations. CFD is an effective means to solve the governing equations (equation $(17,18)$ ) by replacing the integrals/partial derivatives with discretized algebraic forms; thus, the flow field at discrete points in time and/or space can be obtained. The energy equation is neglected in the model since temperatures changes are small.

1) Continuity equation

$$
\frac{\partial \rho}{\partial t}+\nabla \cdot(\rho \vec{V})=0
$$

2) Momentum equation

$$
\begin{aligned}
& \frac{\partial(\rho u)}{\partial t}+\nabla \cdot(\rho u \vec{V})=-\frac{\partial P}{\partial x}+\frac{\partial \tau_{x x}}{\partial x}+\frac{\partial \tau_{y x}}{\partial y}+\frac{\partial \tau_{z x}}{\partial z}+\rho f_{x} \\
& \frac{\partial(\rho v)}{\partial t}+\nabla \cdot(\rho v \vec{V})=-\frac{\partial P}{\partial y}+\frac{\partial \tau_{x y}}{\partial x}+\frac{\partial \tau_{y y}}{\partial y}+\frac{\partial \tau_{z y}}{\partial z}+\rho f_{y} \\
& \frac{\partial(\rho w)}{\partial t}+\nabla \cdot(\rho w \vec{V})=-\frac{\partial P}{\partial z}+\frac{\partial \tau_{x z}}{\partial x}+\frac{\partial \tau_{y z}}{\partial y}+\frac{\partial \tau_{z z}}{\partial z}+\rho f_{z}
\end{aligned}
$$

where $\tau_{i j}$ is the shear stress, $f_{i}$ is the body force per unit mass.

To realize the compressibility of the water, a simplified equation of state (Eq. (1-2)) is applied to pressure-density dependence of the fluid. Moreover, the standard $k-\varepsilon$ turbulence model is selected [16].

\subsection{MOC-CFD model implementation using UDF's}

The key issue in the integration of the MOC and CFD models is to implement the data exchange between the MOC and the CFD modelled components in an RPV system. ANSYS Fluent is used to complete the transient analysis, which allows users to customize specific capabilities using UDFs. Compared with previous models [32-33] that must be compiled on their own platform, all the enhanced applications used in this paper are programmed using $\mathrm{C}$ language and implemented by calling UDFs. This approach makes the coupling of the MOC model and the CFD model more succinct and efficient.

2.4.1 The basic idea for the MOC-CFD model 
The computational domain of an RPV system is divided into two parts, an MOC domain (including the reservoir and a specified length of the pipe) and a CFD domain (including the remaining part of pipe and the valve). As shown in Fig. 2, the reservoir is assumed to be a virtual point, and the valve is modeled as a 3-D CFD model. The two parts of the pipe, the MOC part and the CFD part, are coupled by an overlapping section (transparent blue domain). The interface, shown as a blue point, represents the position of the data exchange between the MOC and the CFD models.

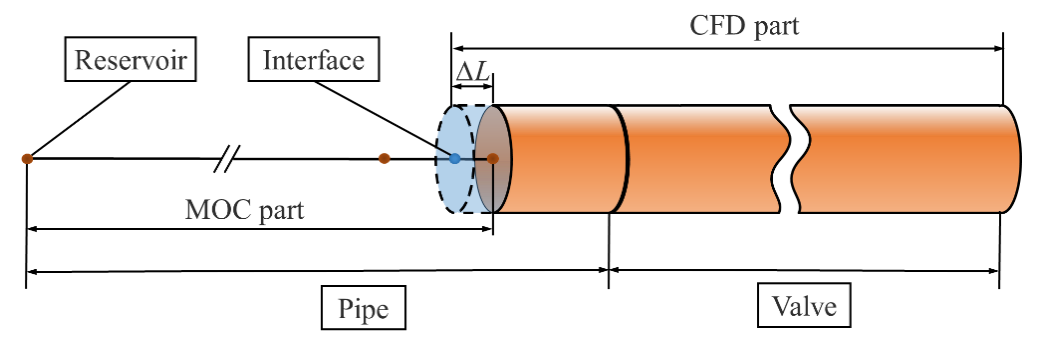

Fig. 2 Schematic of the MOC-CFD model

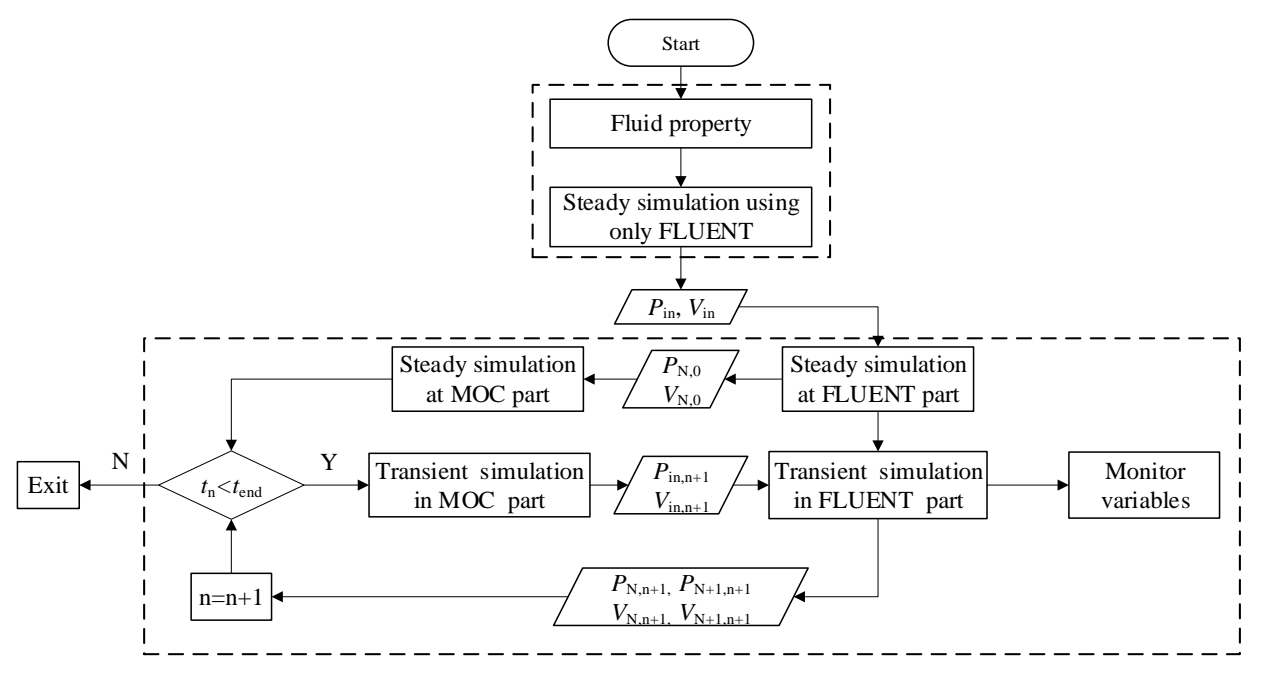

Fig. 3 Flow chart of the MOC-CFD model

The flow chart of this model, shown in Fig. 3 begins with a fluid property setting to realize the compressibility of the fluid. Next, a steady full CFD simulation with a fully opened valve is conducted to obtain the initial flow field. This means that the static pressure and the mean velocity of the interface are used as transfer parameters here, and they are set to be the initial boundary conditions of the CFD part of the new model. A steady simulation based on the MOC- 
CFD model is developed by calling DEMAND UDFs to obtain the initial parameters of the pipe. Next, the transient simulation begins, with the transfer parameters obtained every time step using EXCUTE UDFs and assigned to the interface using PROFILE UDFs at the next time step. During this process, ADJUST UDFs are called to obtain the variation of pressure and velocity at each cross section. Lastly, the transient analysis using the full CFD model is considered to be the benchmark, and the results of the proposed MOC-CFD model are compared with those of the full CFD model to evaluate its accuracy.

\subsubsection{Defining UDFs Using DEFINE Macros}

As mentioned above, several DEFINE Macros are used in the implementation of the MOC-CFD model; some primary Macros are explained herein.

\section{1) DEFINE_EXCUTE_AT_END Macro}

In the transient simulation using the MOC-CFD model, DEFINE_EXCUTE_AT_END macro executes at the end of every time step. The task of this Macro is to complete the calculation of the MOC part at the next step and to obtain the transfer parameters.

Figure 4 is an enlarged detail view of the interface, including an overlapped boundary region (whose length is $\Delta l$ ) that belongs to both the MOC and the CFD domains. It should be noted that the positions of Surface $N$ and $N+1$ are related to the local wave speed (calculated using Eq. (1)), while the wave velocity at the MOC domain is set as a constant, $a_{0}$, to constant values of $\Delta x$. If the time step of the transient simulation is $\Delta t$, then the number of elements in the MOC domain, $N$, can be calculated as

$$
N=I N T\left(\frac{L-L_{C F D}}{a_{0} \Delta t}\right)+1
$$


where $L$ is the length of the pipe, $L_{C F D}$ is the length of the pipe that is modeled as a 3-D model, the "INT" function rounds a number down to the nearest integer. The remaining length of the pipe for the MOC domain can be calculated using $L_{M O C}=N \Delta x$.

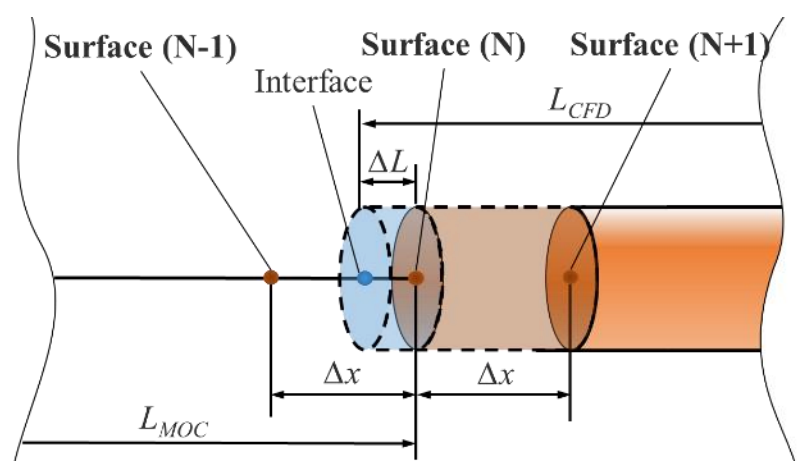

Fig. 4 Details of the interface

Next, the MOC domain is divided into N segments. At every time step, a transient simulation of CFD domain is carried out first, then the variables at Surface N (in the overlapped boundary element) are updated by the latest results. Due to the existence of Surface $(\mathrm{N}+1)$, the flow characteristics along all the MOC computed domain at the next time step are obtained, in which, the mean pressure and velocity at time step $n, P_{i, n}, V_{i, n}(i=0,1,2 \ldots N)$, are obtained using equation (10). The mean pressure and velocity of the interface at time step $n$, namely, $P_{i n, n}, V_{i n, n}$, are obtained through linear interpolation.

$$
\begin{gathered}
P_{i n, n}=P_{N, n}-\frac{\left(L_{M O C}+L_{C F D}-L\right)}{\Delta x}\left(P_{N, n}-P_{N-1, n}\right) \\
V_{i n, n}=V_{N, n}-\frac{\left(L_{M O C}+L_{C F D}-L\right)}{\Delta x}\left(V_{N, n}-V_{N-1, n}\right)
\end{gathered}
$$

These variables are saved as global variables and can be called and recovered at the next time step. The results will be saved to a text document step by step.

\section{2) DEFINE_PROFILE Macro}


DEFINE_PROFILE is used to define the boundary profile as a function of spatial coordinates and time. Generally, the velocity profile of the inlet can be simply set as uniform. But in this case, the inlet boundary for the CFD simulation is actually an interface. As shown in Fig. 5, when the fluid travels through a pipe, a certain distance is required before a fully developed velocity profile is created (as shown in Fig. 6). This distance is called the entrance length and will cause an additional pressure loss (the entrance pressure drop and shown in Fig. 5).

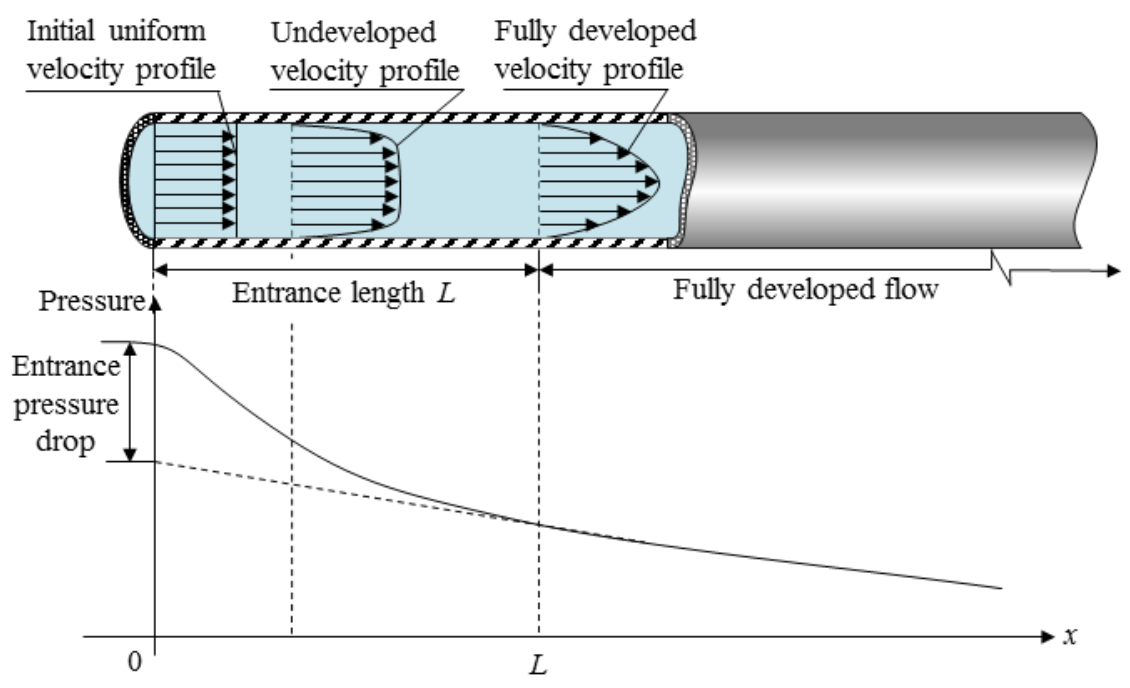

Fig. 5. Developing velocity profiles and pressure changes in the entrance of a duct flow [34]

Thus, an additional error will be introduced if the velocity profile at the data-exchanging interface, shown in Fig. 4 is ignored. To minimize this error, a turbulent velocity distribution function is introduced as follows,

$$
v_{\text {in }}(y)=V_{\text {max }}\left(1-\frac{2 y}{D}\right)^{\frac{1}{n_{e}}}
$$

where $V_{\text {mean }}$ is the mean velocity of the interface, $V_{\max }$ is the maximum velocity at the interface, $y$ is the radial coordinate from the pipe centerline, $v_{i n}$ is the velocity profile at the interface, and $n_{\mathrm{e}}$ is a function of $R e$. The shape of the velocity profile at the cross section of the pipe line is depicted in Fig. 6. The velocities are distributed more uniformly with the increase of $n_{\mathrm{e}}$, and $n_{\mathrm{e}}$ changes with respect to $R e$. 


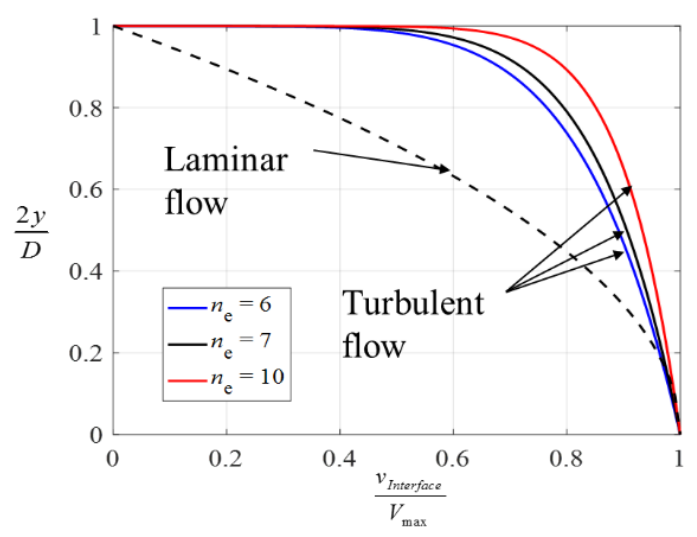

Fig. 6 The shapes of the velocity profile of laminar and turbulent flow [35]

The transfer parameters obtained from the last time step, $P_{i n, n}, V_{i n, n}$, are substituted into the following equations to obtain the flow profile at the interface.

$$
V_{i n, n}=\frac{4 \int_{0}^{\frac{D}{2}} v_{i n}(y) d y^{2}}{D^{2}}
$$

In the steady simulation, DEFINE_PROFILE executes at the beginning of the simulation, and the velocity profile is assigned to the interface through the static pressure $p_{\mathrm{s}}$ and the total pressure $p_{\mathrm{t}}$, according to equation (24). In the transient simulation, DEFINE_PROFILE executes at the beginning of each time step to update the total and static pressure profile of the interface.

$$
\begin{aligned}
& p_{s}(y)=P_{i n, n} \\
& p_{t}(y)=p_{s}(y)+\frac{\rho v_{\text {in }}^{2}(y)}{2}
\end{aligned}
$$

Furthermore, additional DEFINE Macros are used to implement the pressure analysis with the MOC-CFD model. The DEFINE_PROPERTY UDFs are used to specify the compressible material property described in Section 2.1, DEFINE_ON_DEMAND macro is activated after the steady simulation and will be executed immediately to access the transfer parameters from the results of the steady simulation. The DEFINE_ZONE_MOTION macro is executed to specify the valve motion and the DEFINE_ADJUST Macro is used to monitor and access the variables such as the pressure and velocity profile along the pipe. 


\section{VALIDATION}

The multidimensional and multiscale model is validated by comparison with the complete CFD model analysis. The accuracy and efficiency of the multidimensional and multiscale model are evaluated with respect to the friction models and the proportion of the simplified section in the pipe.

\subsection{An RPV system}

A ball valve is widely used in many areas, because of its ease of operation and maintenance; moreover, it has extensive applicability under high pressures and high temperature conditions. As shown in Fig. 7, when the flow passage of the floating ball is in line with the flow (full opening angle), the valve is open; and it is closed when the passage reaches a certain degree with the flow (full closing angle). These processes are implemented by pivoting the lever handle on the stem. It is easy to distinguish the effect of the pressure wave caused by the upstream and downstream boundary condition when the valve is activated. Thus, a reservoir-pipe-ball valve system is investigated herein to understand the interaction between pressure waves and the transient boundary conditions.

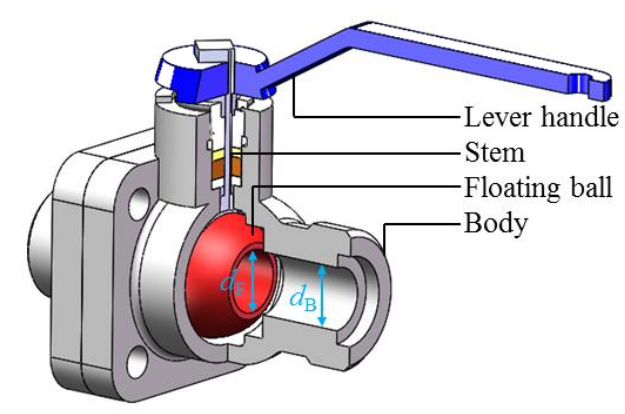

Fig. 7 Cut-away view of ball valve components: 
Table 1 Parameters of the RPV system

\begin{tabular}{ccc}
\hline & Parameters & Value \\
\hline \multirow{2}{*}{ Reservoir } & Total pressure $/ \mathrm{Pa}$ & 1000000 \\
Pipe & Diameter $/ \mathrm{m}$ & 0.02 \\
& Length $/ \mathrm{m}$ & 10 \\
& Inner diameter of the flow passage $\left(d_{\mathrm{F}}, d_{\mathrm{B}}\right) / \mathrm{m}$ & 0.02 \\
& Full opening angle $/{ }^{\circ}$ & 0 \\
valve & Full closing angle $/{ }^{\circ}$ & 83.62 \\
& Pressure outlet $/ \mathrm{Pa}$ & 0 \\
& Closure time $/ \mathrm{s}$ & 0.014 \\
& Closure velocity $/ \mathrm{rad} / \mathrm{s}$ & 100 \\
\hline
\end{tabular}

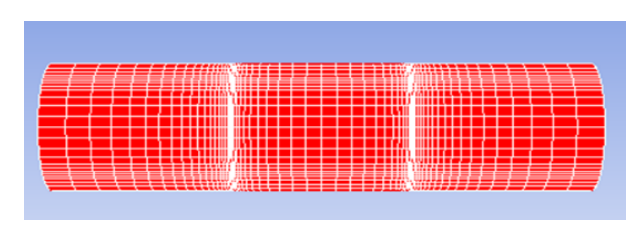

(a)

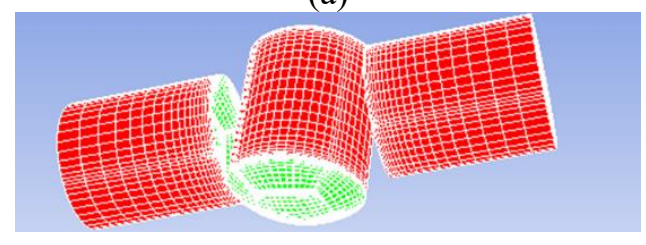

(b)

Fig. 8 Mesh model of the ball valve at different conditions

Table 1 lists the main dimensions of this RPV system and its operating pressure. The total pressure of the system is set as a constant, the valve is initially fully open and a steady state flow of water exists initially. A pressure transient is obtained through a rapid closure of the ball valve in 0.014 seconds (the floating ball is pivoted from $0^{\circ}$ to $80.2^{\circ}$ at a constant speed here to make sure the continuity of the flow field in the CFD simulation). The ball valve is modeled with structured hexahedral meshes to retain more geometry information; its mesh model is shown in Fig. 8, in which (a) shows the opening condition and (b) shows the closing condition. Table 2 shows the grid sensitivity verification 
and the mass flowrate in the RPV system is used to estimate the accuracy of the results. It is found that differences in the mass flowrate between fine mesh and very fine mesh is negligible. Therefore, the fine mesh with $0.2 \mathrm{~mm}$ first layer is sufficient for the transient simulation.

Table 2 The number of grid cells and the corresponding first layer thickness

\begin{tabular}{ccccc}
\hline & Coarse & Medium & Fine & Very fine \\
Thickness of first layer $(\mathrm{mm})$ & 0.8 & 0.5 & 0.2 & 0.1 \\
Number of grid cells & 231,151 & 427,352 & 657,446 & 859,129 \\
Mass flowrate(kg/s) & 3.11 & 3.05 & 3.02 & 3.02 \\
\hline
\end{tabular}

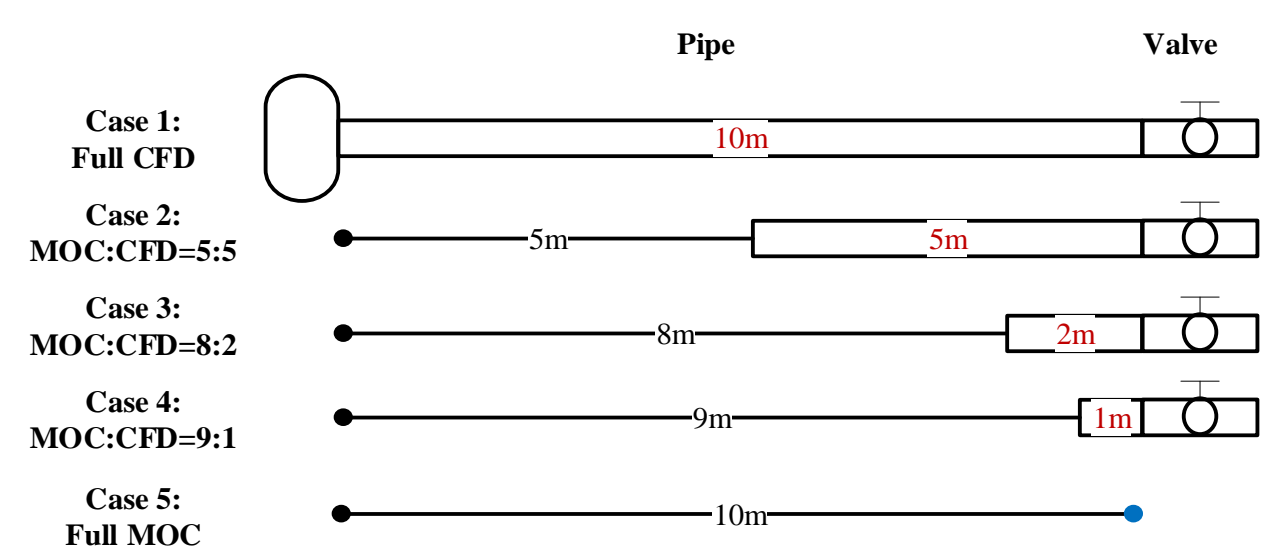

Fig. 9 Schematics of different tested cases

To test the accuracy of this model, five cases were selected with a different fraction of the total pipe length allocated to the MOC or CFD domain. For this investigation, the MOC model uses a steady friction model (SFM), Equ (12), as discussed in section 2.2.2. Schematics of these cases are shown in Fig. 9, where case 1 is the fully CFD modelled case, case 4 is the most simplified using an MOC-CFD approach, and case 5 is the full MOC modelled case (here the ball valve is simplified to be a virtual point, and its flow characteristics is calculated using equation (25)).

$$
V=\frac{C_{d} A_{v} \sqrt{2 \frac{\Delta p}{\rho}}}{A}
$$


where $V$ is the average velocity upstream of the valve, $\Delta p$ is the differential pressure between the upstream and the downstream of the valve, $A$ is the area upstream of the valve, $A_{v}$ is the valve opening area, and $C_{d}$ is the coefficient of discharge [36].

The simplified cases are compared with the full CFD case to validate the feasibility of the proposed model. The metric used to indicate validation is the coefficient of determination, $R^{2}$, illustrating the proportion of the variance in the dependent variable that is predictable from the independent variable. The expression for $R^{2}$ can be written as follows:

$$
R^{2}=1-\frac{\sum_{j}\left(y_{j}-\hat{y}_{j}\right)^{2}}{\sum_{j}\left(y_{j}-\bar{y}\right)^{2}}
$$

where $y_{j}$ denotes the complete CFD model predictions (case 1) for the $j$ time step, $\hat{y}_{j}$ denotes the corresponding prediction for the specific case considered, and $\bar{y}$ is the mean of the complete CFD model responses. $R^{2}$ ranges from 0 to 1 , and a larger value indicates a better accuracy over the whole design space.

\subsection{Results}

Figure 10 shows the mean pressure and the mean velocity along the pipe at $0.005 \mathrm{~s}$ and $0.015 \mathrm{~s}$, respectively. The results with the full CFD case are taken as the benchmark. The flow property simulated using full MOC model has notable differences with that of the full CFD case, and is attributed to the simplification of the ball valve. Figure 11 shows the pressure profile at the symmetry plane and the fluid streamlines in the ball valve at corresponding times in two cases (a full CFD case above and an MOC:CFD=9:1 case below), the results show that even the most simplified case can achieve good predictive accuracy of the flow field. Moreover, with the closing of the valve, the fluid is halted at one side of the valve, causing a rapid increase of the local pressure. The flow pattern inside the valve changes with the operation of the valve, and vortices occur when the flow passage changes. The maximum velocity can increase to $137.19 \mathrm{~m} / \mathrm{s}$ at $t=0.015 \mathrm{~s}$, which is much higher than the average value. 
Therefore, it is difficult to obtain the accurate flow property in the ball valve using a simple function. However, the simulation with a full MOC model (case 5) requires this assumption due to the limitations of the geometric representation. Figure 12 shows the comparisons of pressure and velocity at the end of the pipe (valve end) in the time domain. Similar to the results in the space domain, the trends of the pressure surge in all the simplified cases are consistent except the full MOC case. By comparing the velocity and the pressure in the first $0.02 \mathrm{~s}$, it can be found that the velocity changes during this period of time is larger with the MOC model than that computed using the CFD model, which results in a higher maximum pressure. This overestimation of the maximum pressure in the MOC model may be caused by the introduction of the experimentally determined $C_{d}[1]$, which introduces imprecise opening changes. It is clear that the fluctuation of the pressure in an RPV system, especially the maximum pressure, is sensitive to the valve opening changes. From Fig 12, it is observed that the decrease in pressure amplitude with time is slower when the full MOC is used, suggesting that the energy dissipation is less accurately modelled and that a full MOC model is less desirable.
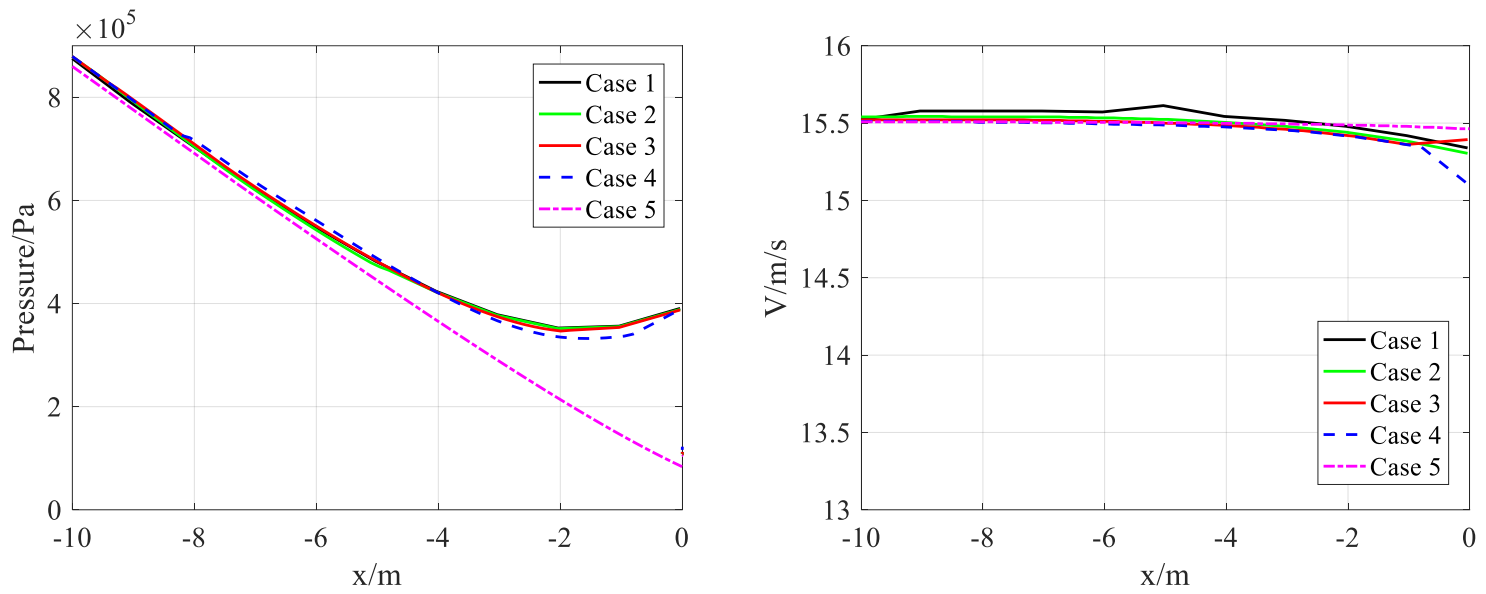

(a) $t=0.005 \mathrm{~s}$ 

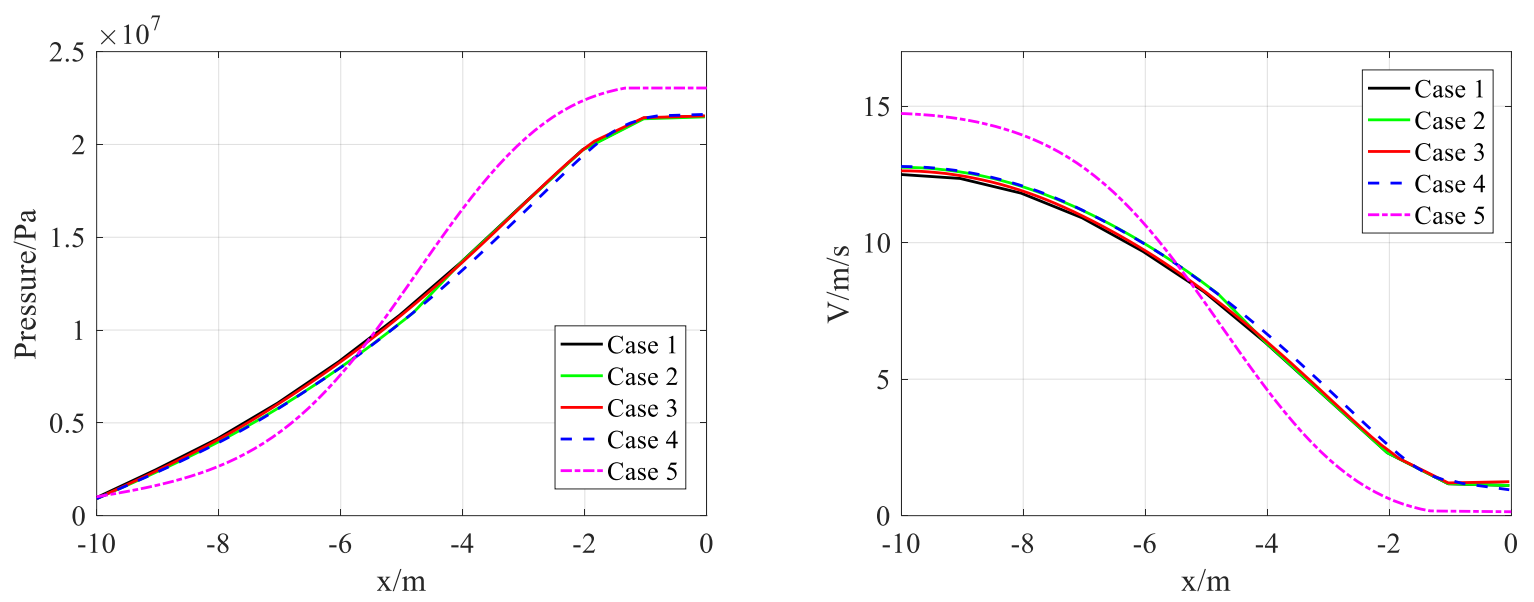

(b) $t=0.015 \mathrm{~s}$

Fig. 10 The mean pressure and velocity along the pipe

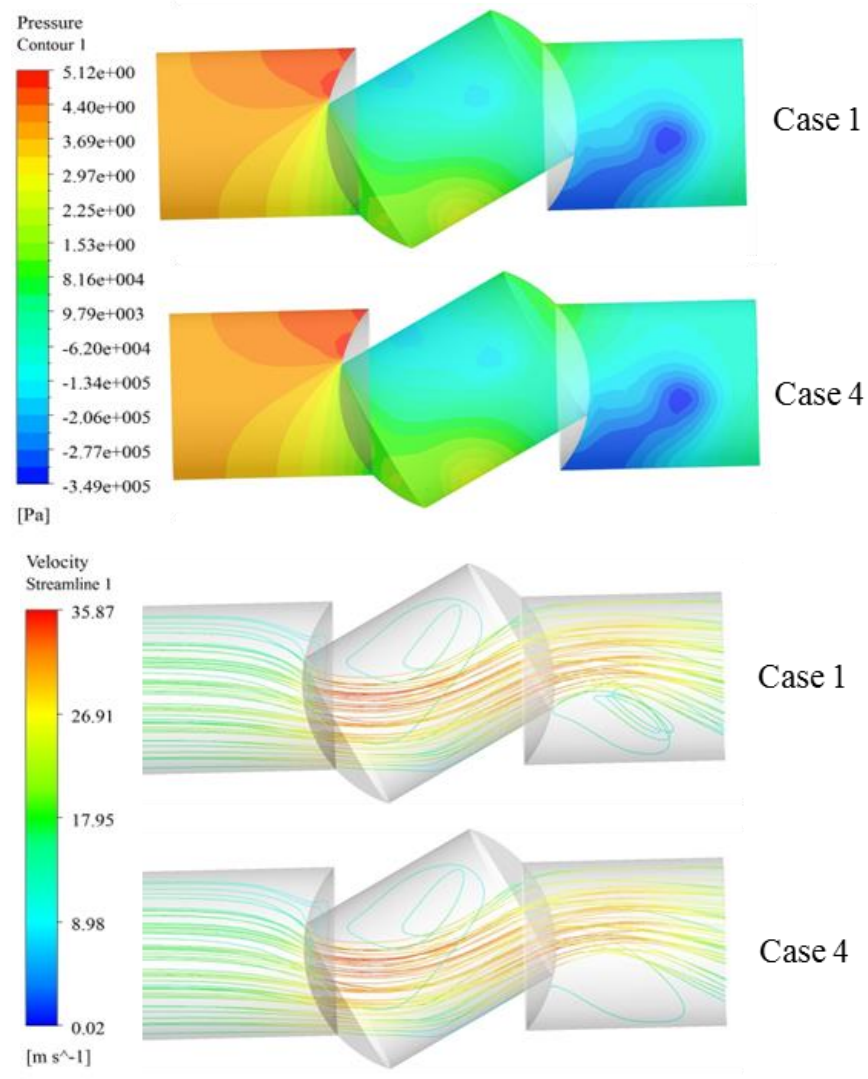

(a) $t=0.005 \mathrm{~s}$ 


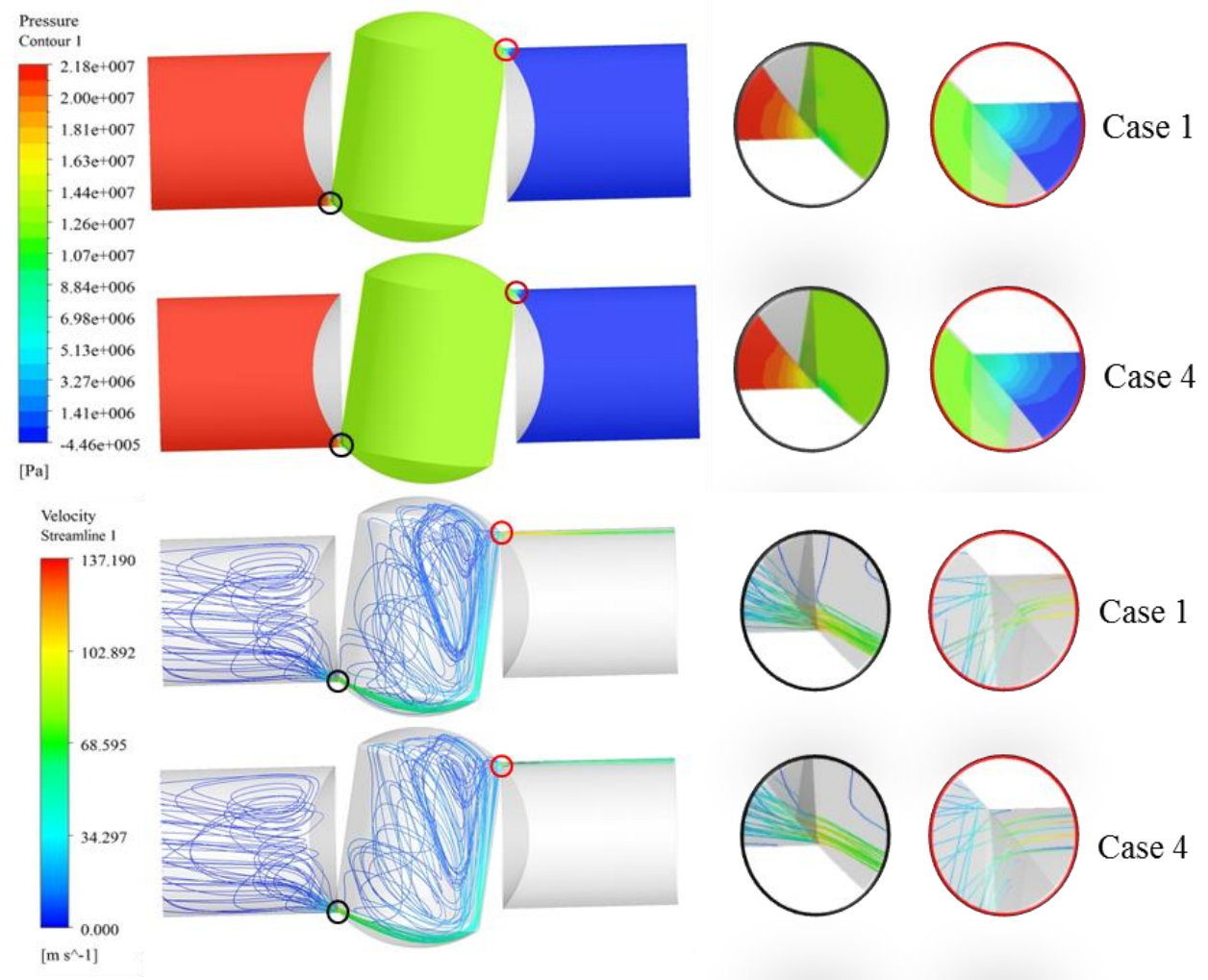

(b) $t=0.015 \mathrm{~s}$

Fig. 11 The pressure contour and streamline at the ball valve
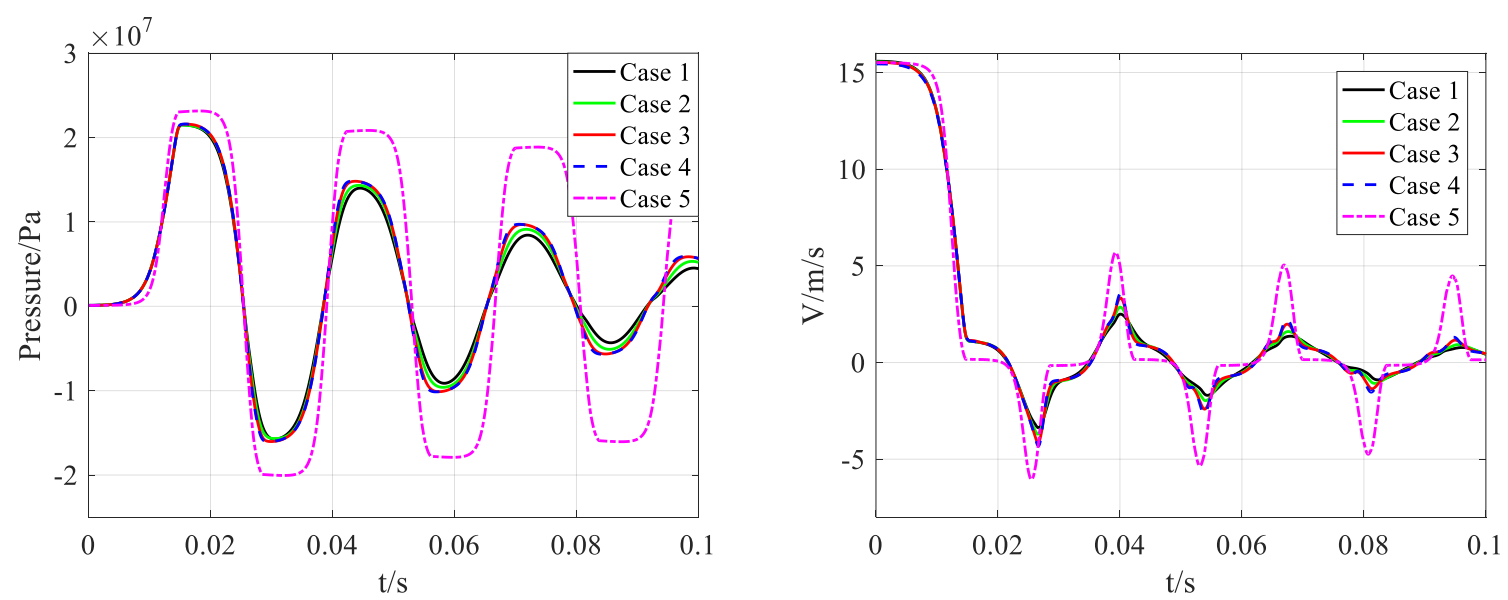

Fig. 12 The mean pressure and velocity at the end of the pipe

The results from the other cases (cases 1-4) suggest that the pressure dissipation with time is smaller in the simplified models compared with that of the full CFD simulation. In addition, it can be observed that the accuracy in every pressure wave cycle decreases with time. Figure 13 shows the accuracy for the first three cycles of the pressure 
waves in different cases compared to the results of the full CFD case (case 1). The values of $R^{2}$ are inversely proportional to time and the length of the MOC domain. The values of $R^{2}$ of Case 4 is reduced to 0.86 , and continuously decreases to 0.18 (Fig. 15) when the pressure waves complete five full periods in the RPV system. This phenomenon is considered to be caused by the use of a SFM approach and uniform velocity profile. Because the SFM and uniform velocity profile have limited accuracy, especially for long transient events, several transient friction models and fully developed velocity profile are tested in the following section.
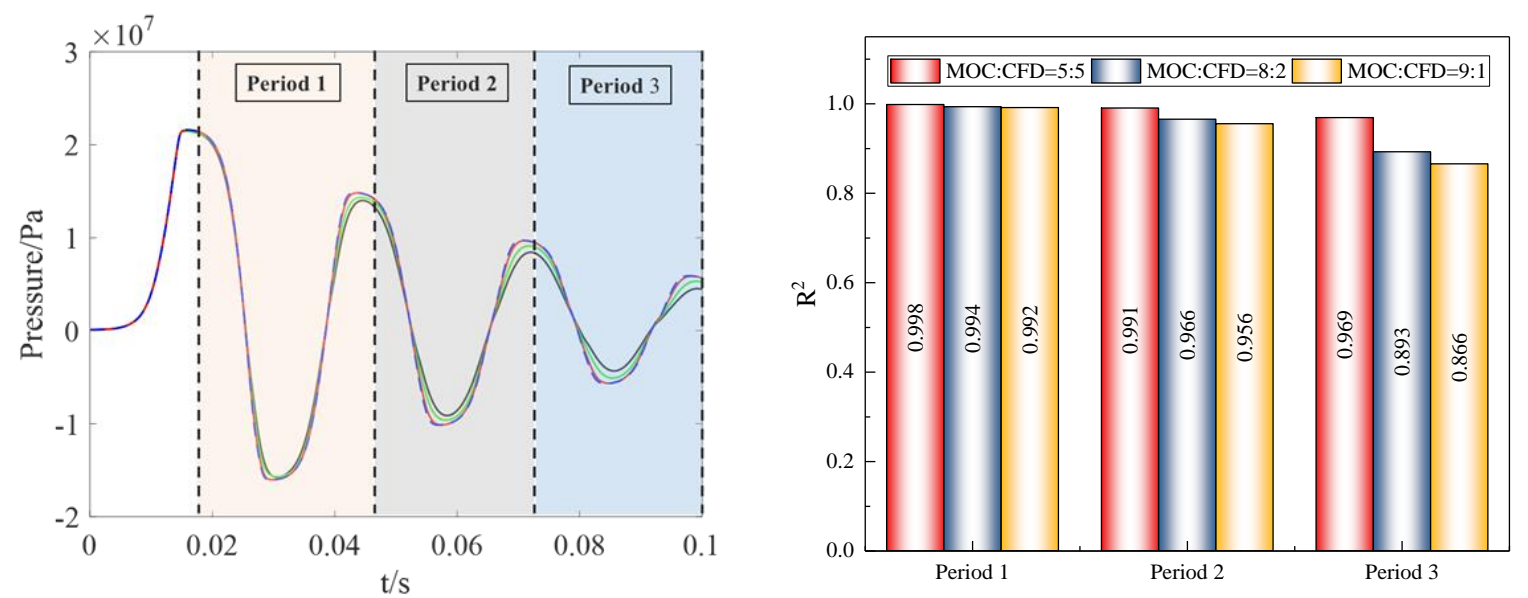

Fig. 13 Accuracy in every period of pressure waves

\subsection{Friction model and velocity profile}

As mentioned in Section 2.2.2, three friction models are tested using Case 4. Here, the pressure variations are regarded as the only variable because the velocity changes with the pressure; thus, only one of them is required to distinguish the trend in the accuracy. The pressure variations at two different positions are shown in Fig. 14 and illustrate that the SFM, IABM, and CIM models give quite similar results; regarding the maximum pressures and wave shape, relatively accurate predictions are obtained. All models slightly overestimate the maximum pressure and the accuracy decreases along with the time; however, the pressure fluctuation is minor after traveling several runs, and a considerable deviation from the reference pressure at this time is acceptable. Nevertheless, the CIM model has 
better agreement in terms of simulating the maximum pressure in every run (Fig. 15), while the computation time is almost the same.

An additional comparison was conducted to assess the effect of the assumed velocity profile. The results with uniform velocity profile and fully developed velocity profile are shown in Fig. 16. From Eq. (26), the accuracy of the simulation results increases from $90.1 \%$ to $96.7 \%$ by considering the velocity profile.

The transient simulations were repeated with the CIM model and fully developed velocity profile for Cases 2-4 and the results shown in Fig. 17, where the accuracy and efficiency for each case in the global time domain is calculated. Even in the most simplified case good accuracy (98.2\%) can be achieved, while its computing time reduces to 0.215 of that required by the complete CFD simulation (case 1 ).

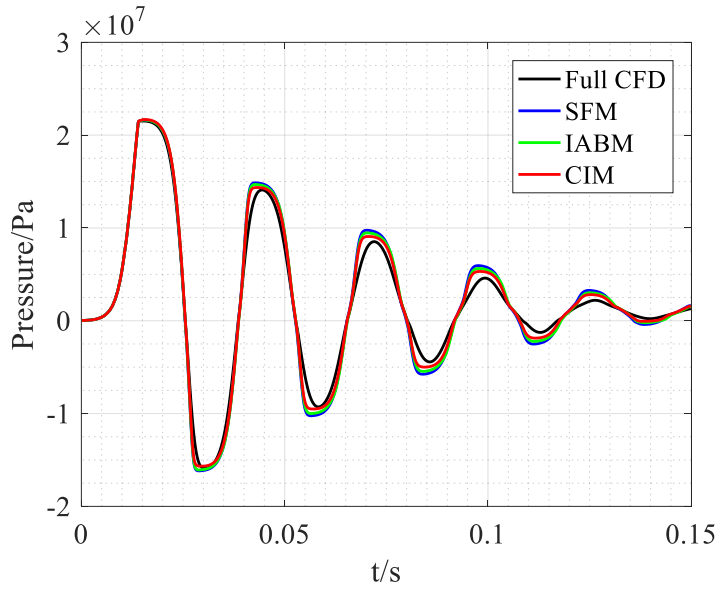

(a) End of pipe

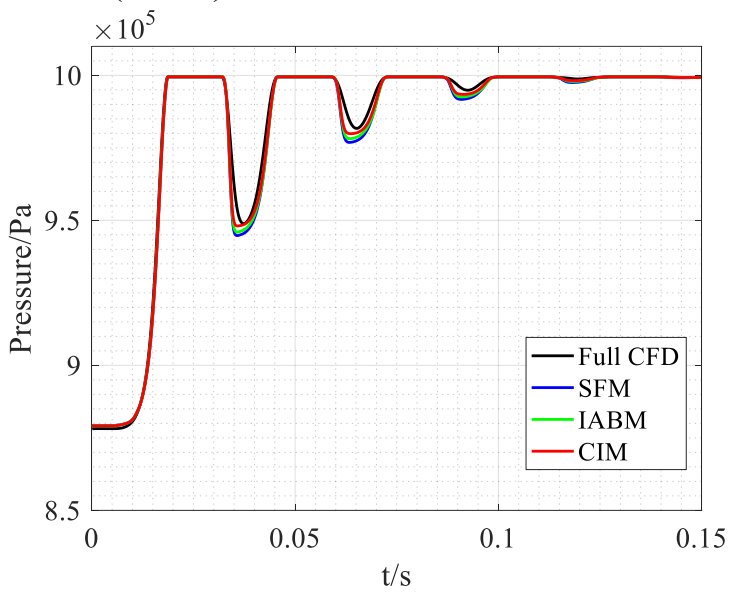

(b) Reservoir

Fig. 14 Pressure variation at the reservoir and the end of the pipe 


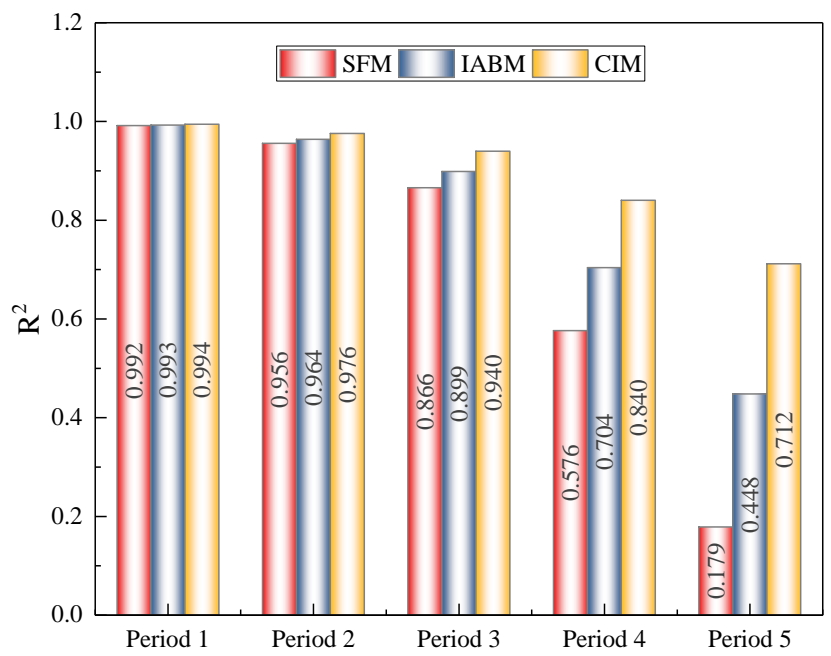

Fig. 15 Accuracy in every period of the pressure waves for different friction models

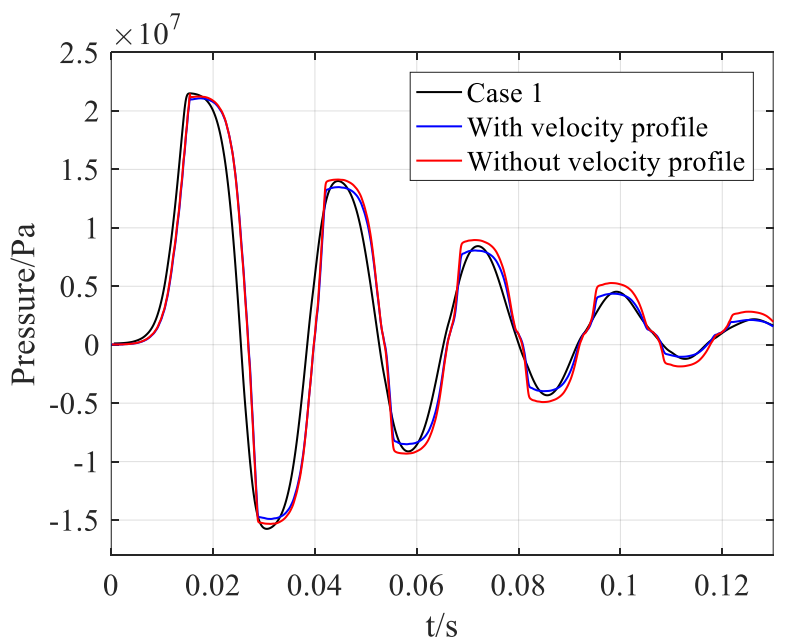

Fig. 16 Pressure variation at the end of the pipe

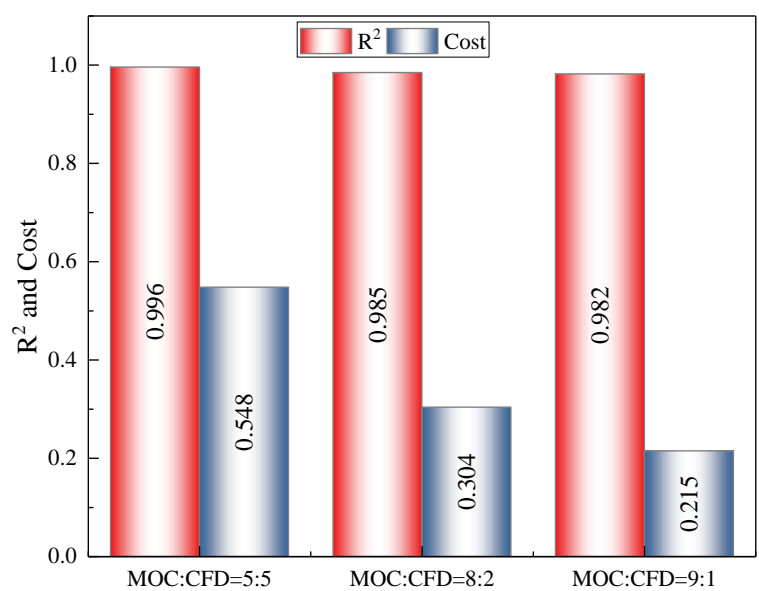

Fig. 17 Accuracy and efficiency in different cases (with CIM friction model) 


\section{PRESSURE ANALYSIS UNDER SOME TYPICAL CONDITIONS}

The multidimensional and multiscale model for Case 4 with CIM has been shown to have good accuracy for the transient analysis of a traditional RPV system. In practice, simplifications, such as constant upstream boundaries and fixed valve-operation curves, are rarely applicable, and the real operating conditions are always accompanied with a disturbance that causes the pressure waves in the RPV system to be disordered and incomprehensible. In this section, the conditions that change the histories of the transient flow are investigated to understand the interaction between the pressure waves and the boundary conditions.

\subsection{Pressure waves}

In a simple valve closing case, it is easy to capture the propagation of the pressure waves. Figure 18 shows the changes in wave shape along the pipeline with time (the dashed lines on the surfaces display the pressure variation at a certain time). A number of points can be made: (1) A series of compression waves are generated during the closure of the ball valve, and the compression wave generated later cannot catch up with the earlier one because of the minimal compressibility of water, and the relatively short pipe; (2) The compression waves propagate towards the upstream direction, and the energy of the pressure waves is dissipated during the propagation process; (3) The upstream boundary is set to be a fixed boundary (a constant value is considered here while a time dependent value is considered in Section 4.2), thus the compression waves propagate towards the upstream reservoir, and then reflect as expansion waves; (4) These expansion waves travel towards the downstream valve and dissipate due to the viscosity of water; (5) The expansion waves reflect as compression waves because the valve at this moment has closed and it can be regarded as a fixed boundary (the expansion waves reflect as expansion waves if the valve is open, as verified in Section 4.2); (6) The reflected waves travel back and forth until the energy is completely dissipated. 


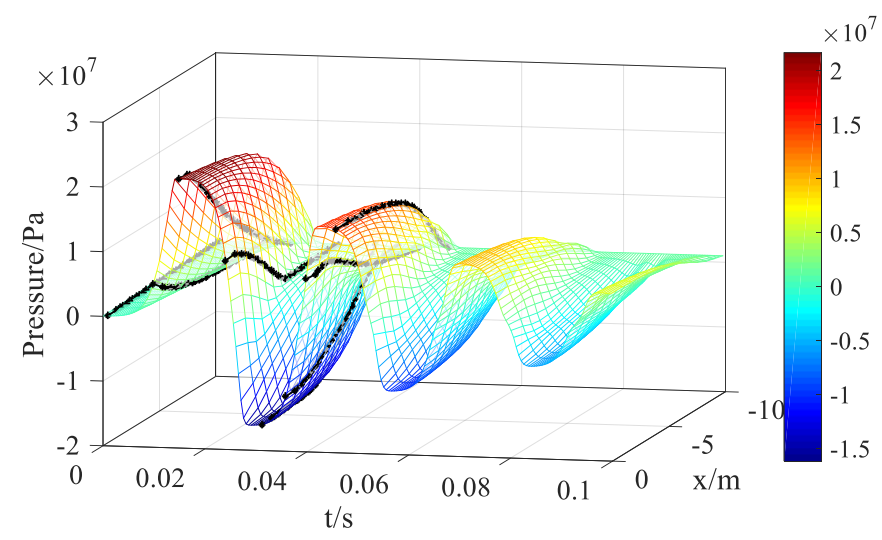

Fig. 18 Wave shape along the pipeline at different times

\subsection{Upstream boundary}

It is commonly assumed that the upstream boundary of an RPV system is at a constant pressure, as is the case for a storage vessel component; however, it is variable if the pressure source is generated by a pump. In this section, the variation of the upstream total pressure, as shown in Fig. 19, is taken into account. The variation of the upstream total pressure is prescribed as a sinusoidal function [37-38], where the amplitude of fluctuation is fixed at $20 \%$ of the mean total pressure (1MPa), and the pump speed is varied resulting in a variation in the period $T_{p}$ of $0.02 \mathrm{~s}, 0.03 \mathrm{~s}$ and $0.04 \mathrm{~s}$. The transient analysis without the closure of the valve is conducted to avoid the effect of pressure surges on the operation of the valve. The fluid characteristics at Surface A and B (shown in Fig. 20) are investigated to assess the propagation of pressure waves in the pipe.

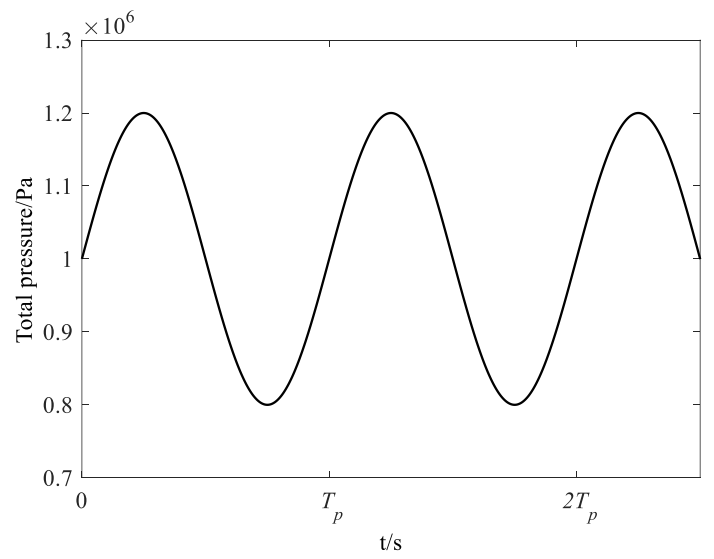

Fig. 19 Variation of the upstream total pressure 


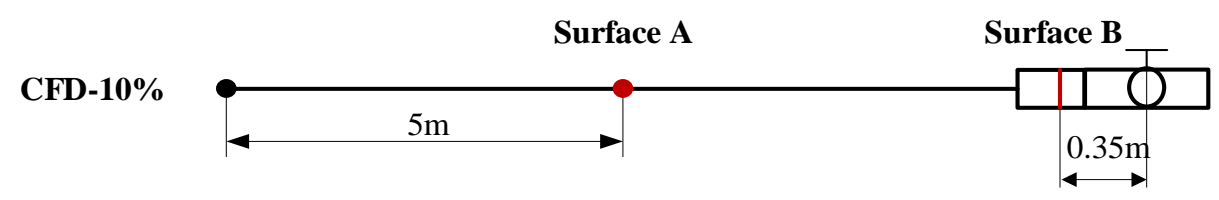

Fig. 20 Positions of Surfaces A and B
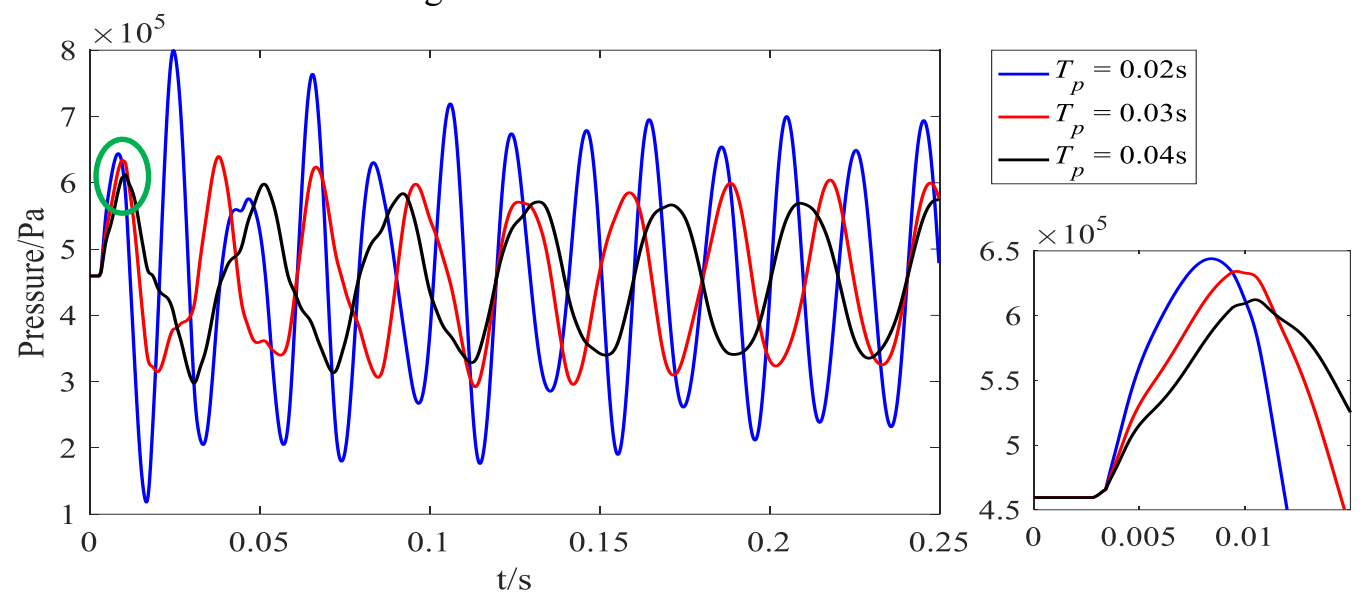

(a) Surface A

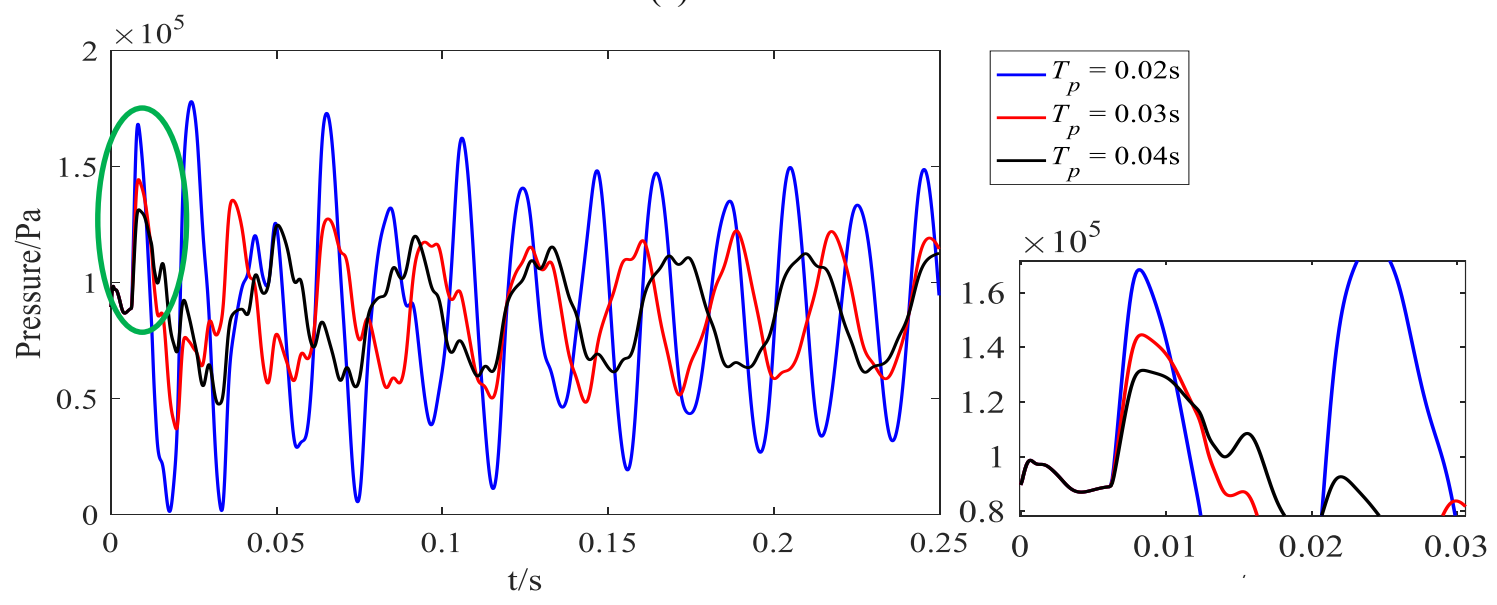

(b) Surface B

Fig. 21 Variation of pressure with different fluctuation periods of upstream total pressure

Figure 21 portrays the pressure surges with different fluctuation periods of the upstream total pressure at Surface A and B; some details of the section highlighted by the green circle are shown in an enlarged view. Note that, with the same fluctuation magnitude, the maximum pressure response is significantly different for each case depending on the source period, especially when $T_{p}$ is $0.02 \mathrm{~s}$. The first crest time of each curve at Surface A is equal to the sum of $T_{p} / 4$ and the time spent in the crest traveling (the traveling distance divided by the sound speed ( about 1483.24 
$\mathrm{m} / \mathrm{s}$ ) is equal to $0.0034 \mathrm{~s}[22])$. The first crest magnitude of each curve decreases with the traveling time, due to the fact that the energy loss increases gradually with time. When $T_{p}=0.02 \mathrm{~s}$, the second crest is higher than the first one, and the third crest is much lower than the others. These findings might be due to an acoustic coupling effect. An FFT analysis of pressure variation is conducted, as shown in Fig. 22. The first crest for each case corresponds to its own fluctuation frequency at the upstream boundary. The other crests have the same frequency for all three cases; thus, these frequencies represent the first- to five-order acoustic resonance frequencies. Note that, the magnitude for the first-order acoustic frequency (drawn in a dark line) is abnormally large, as is the magnitude of the first crest; however, the magnitudes at the other acoustic frequencies have only a slight difference. Thus, it can be deduced that: when the fluctuation frequency of the upstream boundary is close to the acoustic frequency, the pulsation will become much more violent, and a new resonance occurs. The magnitude of the new resonance can be much larger than that of the fluctuation of the upstream boundaries in the pipe line (Fig. 21 (a)), and hence the selection of a suitable fluctuation frequency for the boundary condition and the pipe length (affecting the acoustic resonance frequency) is necessary. In practice the pump speed and pipe length are coupled and need to be suitably matched to minimize excessive pressure transients.

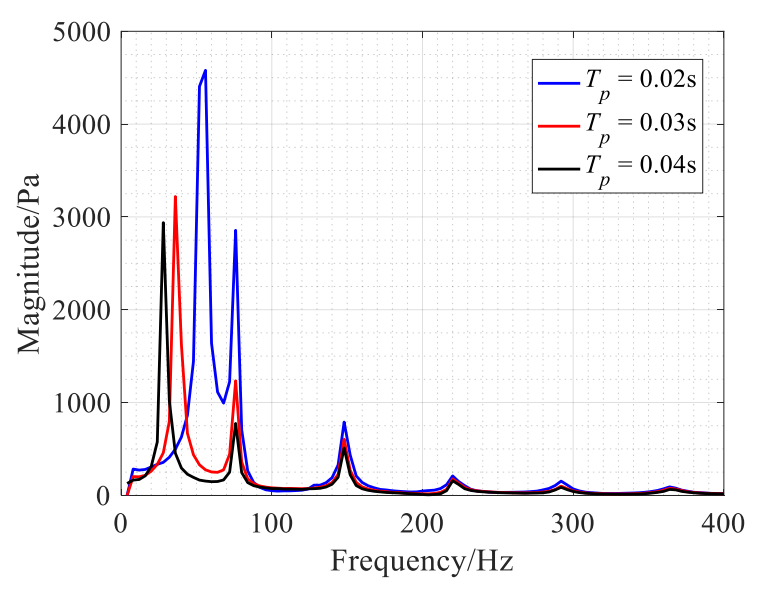

Fig. 22 FFT analysis results of pressure variation at Surface B for different cases

4.3 Downstream boundary 
The closure duration of the ball valve, $T_{c}$, is variable for different situations; clearly, closing the valve more rapidly enhances the pressure waves. Thus, the duration of the ball valve closure affects the maximum pressure. Four cases are considered to evaluate the pressure response in the RPV system, as shown in Fig. 23 where $T_{c}$ is set as 0.07 s, $0.14 \mathrm{~s}, 0.28 \mathrm{~s}$ and $0.7 \mathrm{~s}$. The completely closed position is shown as a dashed line.

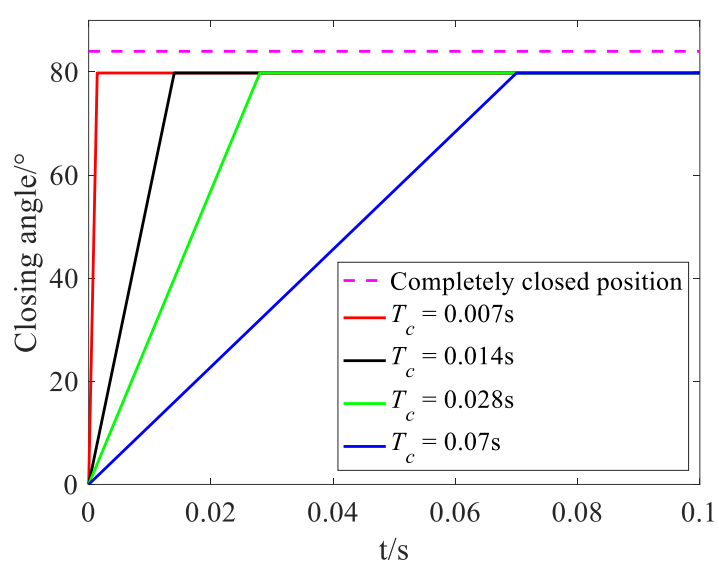

Fig. 23 The valve's opening angle versus time for different cases

The results shown in Fig. 24 verify that a rapid closure of the valve causes a higher maximum pressure. It is well known that when the valve closes rapidly and no reflected compression waves travel back to the valve; the pressure variation is only related to the velocity changes. This is consistent with the results shown in Fig. 24 (b); the maximum pressure remains constant as the value of at a fixed value $T_{\mathrm{c}}$ decreasing from $0.014 \mathrm{~s}$ to $0.007 \mathrm{~s}$. This in turn proves the accuracy of this model.

In addition, an abnormal behavior is observed around the first peak of the pressure (red line Fig. 24 (b), the pressure increases slightly after the valve closure. This behavior occurs because of the existence of fiction in the pipeline, which attenuates the amplitude of the pressure wave fronts. This attenuated value of pressure will recover when the flow velocity in the pipeline decreases to a very small extent. This phenomenon is called line packing [3940]. Because the length of the pipeline tested in this paper is $10 \mathrm{~m}$, the attenuation is small compared with the sudden pressure increase caused by the valve closure. In other cases, the amplitude of the reflected expansion waves are much larger than the recovering values, and line packing cannot be observed in these cases. 

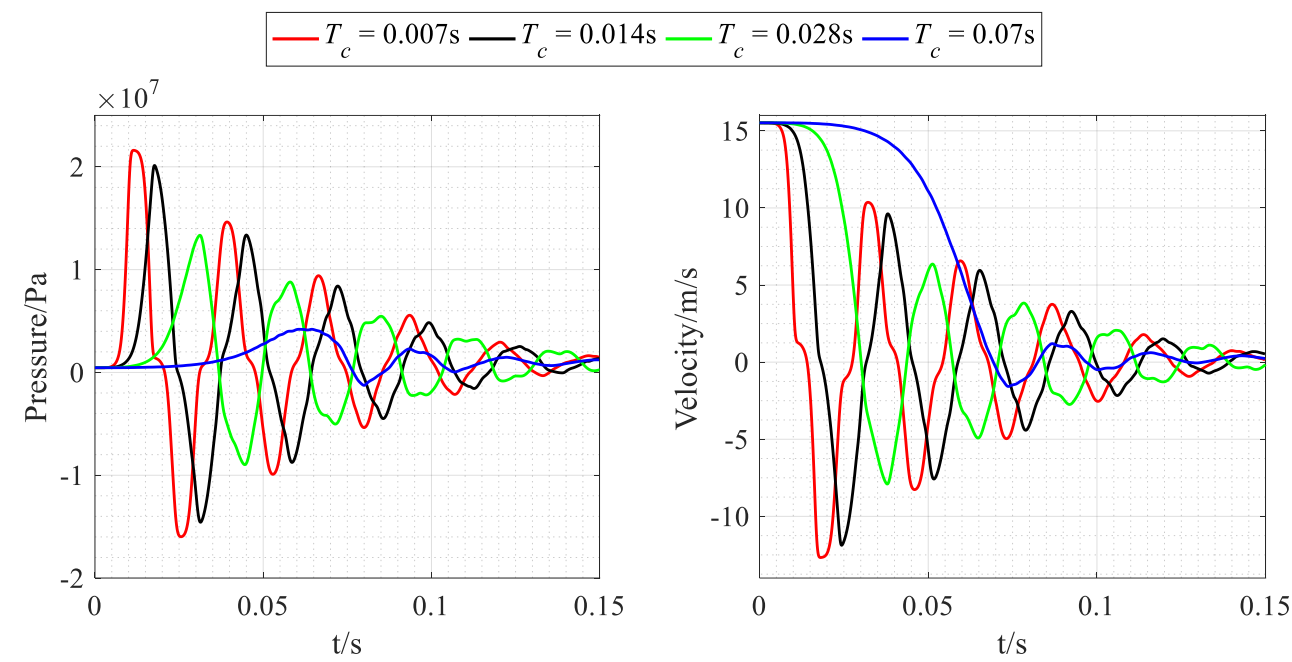

(a) The variation of pressure and velocity at Surface A

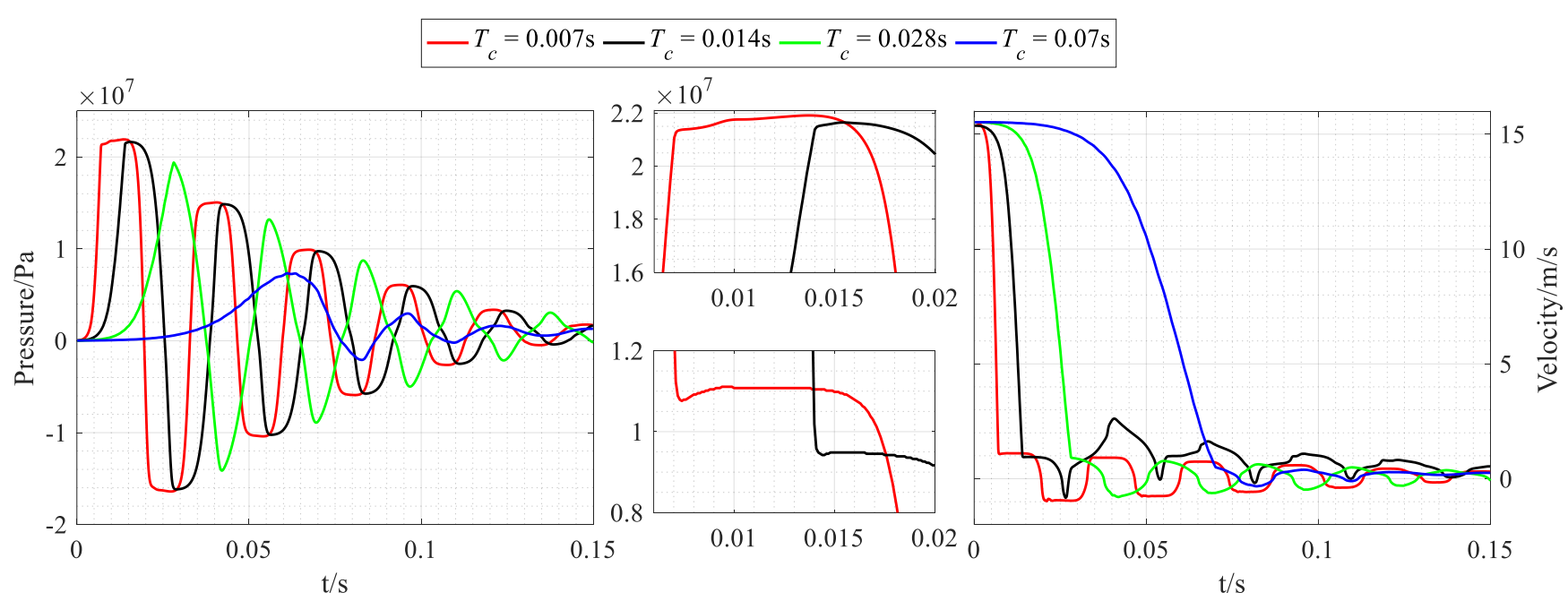

(b) The variation of pressure and velocity at Surface B

Fig. 24 The variation of pressure at different points for different cases

\section{CONCLUSION}

A multidimensional and multiscale model of an RPV system is proposed by combining the 1-D MOC model of the connecting pipe and the 3-D CFD model of a ball valve. Based on this model, a pressure analysis was conducted to examine the propagation of the pressure waves and their coupling phenomenon with the boundary conditions. The conclusions are as follows: 
(1) Due to the reliance on empirical formulas, the simulation with full MOC model cannot provide a highly accurate simulation.

(2) This MOC-CFD model presented in this paper has no adjustment in the pipe length or the wave speed, and considers the velocity profile at the data exchanging interface. The simulation with a coupled MOC-CFD model results in a significant improvement of the efficiency by decreasing the computing time to $21.5 \%$ of that for the full CFD model, while the accuracy is higher than $98 \%$.

(3) The friction model is important in the prediction of the pressure response, especially for long time prediction. The results show that the CIM friction model is more suitable in this case.

(4) The variation at an upstream boundary causes persistent pressure fluctuations in the system. The coupling of the upstream fluctuation and the acoustic resonance in the system will result in a more violent pressure fluctuation.

The high fidelity predictions of this MOC-CFD model is validated using a simple reservoir-pipe-ball valve system, which makes it possible to obtain the accurate response in a more complex system. With this model, passive valves, pumps and other geometrically complex components, which are difficult to simplify, can still be calculated using three-dimensional CFD models, while the other parts are simulated using one-dimensional MOC models. This MOC-CFD approach allows accurate prediction of the transient flow characteristics in the system, and extension of the techniques to validate the performance of components under actual working conditions.

\section{ACKNOWLEDGMENTS}

This work was supported from The National Natural Science Foundation of China (No. 51505061, No. U1608256).

\section{REFERENCES}

1. Streeter, V. L., and L. Sou. 1993. Fluid Transients in Systems. Prentice-Hall, Englewood Cliffs, New Jersey. pp. 21-65. 
2. Tijsseling, A. S., and. Lavooij C. S. W. 1990. Waterhammer with Fluid-Structure Interaction. Applied Scientific Research 47(3), 273-85.

3. Vardy, A.E., and Tijsseling A.S.. 2016. Method of Characteristics: Why Is It so Good? BHR Group - 12th International Conference on Pressure Surges (February).

4. Tijsseling, A. S. 2003. Exact Solution of Linear Hyperbolic Four-Equation System in Axial Liquid-Pipe Vibration. Journal of Fluids and Structures 18(2): 179-96.

5. Tijsseling, A.S. 1996. Fluid-Structure Interaction in Liquid-Filled Pipe Systems: A Review. Journal of Fluids and Structures 10(2): 109-46.

6. Johnston, Nigel, Min Pan, Sylwester Kudzma, and Pengfei Wang. 2014. Use of Pipeline Wave Propagation Model for Measuring Unsteady Flow Rate. Journal of Fluids Engineering 136(3): 031203.

7. Xu, Yuanzhi, and Zongxia Jiao. 2017. Exact Solution of Axial Liquid-Pipe Vibration with Time-Line Interpolation. Journal of Fluids and Structures 70(November 2016): 500-518.

8. Ferràs, David, Pedro A. Manso, Anton J. Schleiss, and Dídia I.C. Covas. 2016. Fluid-Structure Interaction in Straight Pipelines: Friction Coupling Mechanisms. Computers and Structures 175: 74-90.

9. Song, X. G., Wang, L., and Park, Y. C. 2010. Transient analysis of a spring-loaded pressure safety valve using computational fluid dynamics (CFD). Journal of pressure vessel technology 132(5): 054501.

10. Song, X. G., et al. 2014. A CFD analysis of the dynamics of a direct-operated safety relief valve mounted on a pressure vessel. Energy Conversion \& Management 81(2): 242-246.

11. Song, X. G., Wang, L. T., Park, Y. C., and Sun, W. 2015. A fluid-structure interaction analysis of the springloaded pressure safety valve during popping off. Procedia Engineering 130: 87-94.

12. Hős, C., Bazsó C., and A. Champneys. 2015. Model reduction of a direct spring-loaded pressure relief valve with upstream pipe. IMA Journal of Applied Mathematics 80(4): p. 1009-1024. 
13. Yang, L., et al. 2017. Experiments and transient simulation on spring-loaded pressure relief valve under high temperature and high pressure steam conditions. Journal of Loss Prevention in the Process Industries 45: 133146.

14. C. J. Ye Qifang. 2009. "Dynamic analysis of a pilot-operated two-stage solenoid valve used in pneumatic system," Simulation Modelling Practice and Theory 17, 794-816,

15. Darby, R. and A.A. Aldeeb. 2014. The dynamic response of pressure relief valves in vapor or gas service. Part III: Model validation. Journal of Loss Prevention in the Process Industries 31: 133-141.

16. Yang S., Chen X., Wu D., et al. Dynamic analysis of the pump system based on MOC-CFD coupled method. Annals of Nuclear Energy, 2015, 78: 60-69.

17. Wang C., Håkan Nilsson, Yang J., et al. 1D-3D coupling for hydraulic system transient simulations. Computer Physics Communications, 2016, 210: 1-9.

18. Mandair, S., Karney, B., Magnan, R., Morissette, J.-F. 2018. Comparing Pure CFD and 1-D Solvers for the Classic Water Hammer Models of a Pipe-Reservoir System. 1st International WDSA / CCWI 2018 Joint Conference, Kingston, Ontario, Canada, July 23-25, 2018.

19. Galindo, J., Tiseira, A., Fajardo, P., and Navarro, R. 2011. Coupling Methodology of 1D Finite Difference and 3D Finite Volume CFD Codes Based on the Method of Characteristics. Math. Comput. Model., 54(7-8): 17381746.

20. Fajardo, D. P. 2012. Methodology for the Numerical Characterization of a Radial Turbine under Steady and Pulsating Flow. Ph.D. Thesis, Universitat Politècnica de València.

21. ANSYS, Inc. ANSYS 18.0 Help. ANSYS, Inc. 2016.

22. Chaudhry, M. H. 2014. Applied Hydraulics Transients. Springer-Verlag, New York. pp. 55-108.

23. Vardy, Alan E. et al. 1992. A Characteristics Model of Transient Friction in Pipes. Journal of Hydraulic Research 30(4): 569-70. 
24. Brunone, B., Golia, U. M., \& Greco, M. 1991. Some Remarks on the Momentum Equation for Fast Transients. International Meeting on Hydraulic Transients with Water Column Separation (9th Round Table of the IAHR Group): 201-209.

25. Ramos, Helena, Dídia Covas, Alexandre Borga, and Dália Loureiro. 2004. Surge Damping Analysis in Pipe Systems: Modelling and Experiments. Journal of Hydraulic Research 42(4): 413-25.

26. Zielke, W. 1968. Frequency-Dependent Friction in Transient Pipe Flow. Journal of Basic Engineering 90(1): 109 .

27. Trikha, A. K. 1975. An Efficient Method for Simulating Frequency-Dependent Friction in Transient Liquid Flow. Journal of Fluids Engineering 97(1): 97.

28. Vardy, A. E., and Kuo-Lun Hwang. 1993. A Weighting Function Model of Transient Turbulent Pipe Friction. Journal of Hydraulic Research 31(4): 533-48.

29. Vardy, A. E., and J. M.B. Brown. 2004. Transient Turbulent Friction in Fully Rough Pipe Flows. Journal of Sound and Vibration 270(1-2): 233-57.

30. Haaland, S.E. 1983. Simple and Explicit Formulas for the Friction-Factor in Turbulent Pipe Flow, Transactions of the ASME, Journal of Fluids Engineering 105(1), 89-90.

31. Anderson Jr., J.D., 1995. Computational Fluid Dynamics: The Basics with Applications. Mc-Graw.: 3-93.

32. Yang Shuai, Xin Chen, Dazhuan Wu, and Peng Yan. 2015. Dynamic Analysis of the Pump System Based on MOC-CFD Coupled Method. Annals of Nuclear Energy 78: 60-69.

33. Wu, Dazhuan, Shuai Yang, Peng Wu, and Leqin Wang. 2011. MOC-CFD Coupled Approach for the Analysis of the Fluid Dynamic Interaction between Water Hammer and Pump 141(6): 1-8.

34. Munson, B. R., Okiishi, T. H., Hubsch, W. W., and Rothmayer, A. P. 2013. Fundamentals of Fluid Mechanics, 7th ed. John Wiley \& Sons, Inc., New York. pp. 400-431.

35. White, F. M. 2009, Fluid Mechanics, 6th ed. McGraw-Hill College. pp. 347-360. 
36. Wang D, Bai C. 2018. The Parametric Modeling of Local Resistance and Pressure Drop in a Rotary Ball Valve. ASME. J. Fluids Eng. 140(3): 0-11.

37. Nakamura, T., et al. 2013. Flow-Induced Vibrations: Classifications and Lessons from Practical Experiences. Academic Press. pp. 197-275.

38. Xu Zhenyu, Miao Xuhong, and Zuo Hai. 2015. The Research on Pulsation of Pump Pressure in Water Mist System. Energy Procedia. 66: 73-76.

39. Wahba EM. 2016, On the Propagation and Attenuation of Turbulent Fluid Transients in Circular Pipes. ASME. J. Fluids Eng. 138(3): 0-7.

40. Liou JP. 2016. Understanding Line Packing in Frictional Water Hammer. ASME. J. Fluids Eng. 138(8): 06. 